\title{
Physics Essays
}

\section{AN INTERNATIONAL JOURNAL DEDICATED TO FUNDAMENTAL QUESTIONS IN PHÝSICS}

Note by Jack Sarfatti March 25, 2017

The device will not work. Stapp was right. The linearity and unitarity of QM forbids locally decodable keyless entanglement messaging (aka signaling). PQM with Roderick Sutherland's wave action $<->>$ particle reaction post-Bohmian Lagrangian is a nonlinear non-unitary nonstatistical locally retrocausal weak measurement theory that does allow what we were looking for in this paper.

"Little could Herbert, Sarfatti, and the others know that their dogged pursuit of faster-than-light communication -and the subtle reasons for its failure-would help launch a billion-dollar industry. ... Their efforts instigated major work on Bell's theorem and the foundations of quantum theory. Most important became known as the "no-cloning theorem," at the heart of today's quantum encryption technology" MIT Physics Professor David Kaiser in the book "How the Hippies Saved Physics"

PUBLISHED BY UNIVERSITY OF TORONTO PRESS FOR ADYANCED IASER AND FUSION TECHNOLOGY, INC.

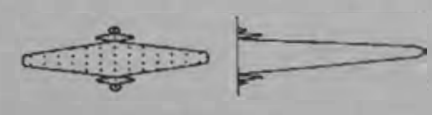




\title{
Design for a Superluminal Signaling Device
}

\section{Ielk Sertanti}

\begin{abstract}
A gedanken experiment design for a superluminal (faster than light - FTL) signaling device using polarization-correlated photon pairs emitted back-to-back is given. Only the Feymman rules for standard quantum mechanics are used. The new approach is to let the transmitter pboton interfere with itself. It is then passible to transmit and locally decode superluminal signals by the controllable shifting of quantum photon polarization probabilities acrass arbitrary space-time separations between the detections of the two photons in the same pair. The superluminal signal is encoded with the message by rotaling a calcite interferometer transmitter detector relative to a fixed receiver calcite detector through angle $\theta$ or, alternatively, by changing the average interferometer phase $\phi_{0}$. The degree of polarization of the light at the receiver detector depends upon these tuo parameters, $\theta$ and $\phi_{0}$. Therefore, the message is decoded by monitoring the changing degree of linear polarization of the receiver light. The principle of causality that effects are always after causes in all frames of reference will be disproved if this derice works as predicted. The objections of causal paradoxes and proofs forbidding superluminal signaling are addressed. The second part of the paper begins a study of the effect of causality violation on the rest of physics. For example, passible violations of the spin-statistics connection and local Lorentz invariance by dark matter, a relativistic quantum time operator, a new view of infinities in quantum field theory, traversable wormboles as time machines, and quantum spin thermodynamics of the mind-matter interaction are some of the topics discussed.
\end{abstract}

Key words: superluminal signaling, causality-violating relativity, time travel paradoxes, quantum consciousness

\section{CANTUM INTERFERENCE COMMUNICATION}

bence proceeds as if the past were the home of explanation; whereas tare, and the future alone, holds the key to the mysteries of the pasent

Fenry Dwight Sedgwick, "House of Sorrow," An Apology for Old Maids (1908)

sigpose there is even something vaguely teleological about the effects 4 ursciousness, so that a future impression might affect a past action. Roger Penrose, F.R.S., The Emperor's New Mind ${ }^{1}$

Z exms to me that biological systems are able in some way to utilize te coposite time-sense in which radiation propagates from future to zes. Bizzare as this may appear, they must somehow be working riards in time.

Sir Fred Hoyle, F.R.S., The Intelligent Universe (Endnote 2, p. 213).

to the reader. The point of view about the nature of physics that I here is controversial, not respectable in either style or substance in zicitional academia, spawned in the romantic Caffe Trieste of San Fran- cisco's Bohemia (North Beach), eccentric and beyond the cutting edge of, for example, Paul Davies' excellent collection New Pbysics. Nevertheless, the referee has courageously written: "The actual gedankenexperiment is for superluminal information transfer of a type different from the FLASH proposal of Nick Herbert. Although I intuitively agree with Stapp's feelings (referred to in the paper), the design is significantly different from Herbert's and has a contrihution of value in the documentation of possible designs of superluminal signalling devices which can be subject to discussion and criticism by the physics community. Thus, although I think that this design will probably suffer the same fate as Herbert's FLASH, I believe it should be published in a form which makes it available for critical scrutiny and discussion. This process either results in a new potential device or helps to complete the case against such devices through the subsequent discussion, as Herbert's example in Foundations of Pbysics has done."

I am predicting a startling new phenomenon, hitherto thought to be impossible in principle - the distant and/or retroactive control of polarization of photons (and spin of massive particles) via nonlocal quantum correlations as the communication channel. My essential intuition in the following qnantitative model is not difficult to grasp. In the simplest case, for two pho- 
tons 1 and 2 emitted back-to-back, let the two doubly refracting crystals be perfectly aligned. The results of linear polarization $(V$ and $H$ ) measurements, when neither photon is allowed to interfere with itself (Fig. 1), are computed from the pure spin-entangled pair state $\left[|V\rangle_{1}|V\rangle_{2}+|H\rangle_{1}|H\rangle_{2}\right] / \sqrt{ } 2$. The distant correlations of photon 1 to orthogonal spin states of photon 2 ensure that photon 1 is completely unpolarized in local measurements. Now, on the other hand, suppose (in analogy with the well-known spin-flip technique in one path of a neutron interferometer, and the Berry-Chiao phase technique using a coiled optical fiber that adiabatically rotates the plane of polarization of a photon) that we can coherently and adiabatically disentangle the above pair state without collapsing it into the new form $\left[|V\rangle_{1}|V\rangle_{2}+|H\rangle_{1}|V\rangle_{2}\right] / \sqrt{ } 2=\left[|V\rangle_{1}+|H\rangle_{1}\right]|V\rangle_{2} / \sqrt{ } 2$. We do this by letting photon 2 interfere with itself and rotating its polarization by $90^{\circ}$ in one of the two interfering paths (Fig. 3). The two photons are no longer spin-correlated. Each has its own spin state. But now, photon 1 is completely polarized at $45^{\circ}$ to the common orientation of both aligned crystals. Compare this new situation to the old one where photon 2 did not interfere with itself - a cboice which forced photon 1 into a mixed unpolarized density matrix with one bit of positive entropy. On the other hand, when we choose to make photon 2 interfere with itself in concert with the adiahatic rotation of polarization in one branch of the interferometer, we have then made the opposite choice which forces photon 1 into a pure polarized zero-bit entropyless state. That is, the choice of forcing photon 2 to interfere with itself properly transmits a negative entropy bit of information to photon 1 and its measuring apparatus. Furthermore, the metric space-time interval between the choice for photon 2 (which is the cause) and the polarization for photon 1 (which is the effect) is irrelevant. The nonlocal quantum spin geometry is premetrical. The space-time interval between active cause and passive effect can be spacelike corresponding to superluminal communication, or it can be arranged to be timelike with delayed cboice in which the cause is in the frame-invariant future of the effect. Indeed, there is a conflict with the axiom of causality of relativity, although not with the classical tests of time dilation and velocity-dependent mass.

In Ref. 1 Mermin discusses recent work by Greenberger, Horne, and Zeilinger $(\mathrm{GHZ})$ on three-particle correlations that go beyond the two-particle correlations of Einstein, Podolsky, and Rosen (EPR) as extended by Bohm and Bell. This new theoretical work provides the basis for a crucial experiment that can show the existence (or nonexistence) of what Einstein called "spooky action-at-a-distance" without a statistical analysis of many measurements. Mermin ${ }^{(2)}$ writes, "Thus in one simple version of the two-particle EPR experiment the hypothesis of elements of reality [i.e., local causality] requires a class of outcomes to occur at least $55.5 \%$ of the time, while quantum mechanics allows them to occur only $50 \%$ of the time. In the $\mathrm{GHZ}$ experiment, on the other hand, the elements of reality [i.e., local causality] require a class of outcomes to occur all of the time, while quantum mechanics never allows them to occur." There is nothing wrong with the EPR criterion of reality "If, without in any way disturbing a system, we can predict with certainty the value of a physical quantity then there exists an element of physical reality corresponding to this physical quantity." What is probably false-to-fact is the assumption "without in any way disturbing the system." In addition, there is the problem that if quantum action at a distance is real, can it be controlled within standard quantum mechanics? That is the main focus of this paper.

Josephson $^{(3)}$ suspects that living matter does manage to control quantum nonlocality, but in a way that is beyond the formal structure of present- day quantum mechanics. I agree that living matter probably does control quantum nonlocality, but that the way in which it does so can be understood using present-day quantum mechanics. What has to be profoundly modified, in my view, is not quantum mechanics, but the causal axiom of relativity without ahandoning the fundamental symmetry of relativity that the laws of nature should be independent of the frame of reference.

\subsection{New Kinds of Signals?}

The nonstandard distinction between signals of the first, second, and third kind is developed in more detail in Sec. 2 of this paper. However, briefly, I adopt the modern fiber bundle extension of world geometry as used in the gauge theory of fundamental forces. Subluminal and luminal signals of the first kind obey causality and involve decodable energy flows inside and on the light cone, respectively. Superluminal and retroactive signals of the second kind violate causality in a "globally self-consistent" way ${ }^{3}$ and involve decodable energy flows outside the light cone, and similarly for signals of the third kind, which the following gedanken experiment purports to generate. For causality-violating signals of the third kind, the communication channel is the controllably nonlocal quantum correlations in the extra dimensions of internal fiber spaces beyond space-time. There is now compelling experimental evidence for the breakdown of causality in the dispersion relations for the scattering of gamma photons off protons. ${ }^{4}$ I claim that controllahle nonlocality is part of standard quantum mechanics and that proofs stating otherwise are incorrect. Signals of the third kind will, of necessity, involve energy flows; in the present case they are on the light cone, but these flows within four-dimensional space-time are not the channel where the information is encoded transmitted and decoded. The channel is in the extra nonmetrical "fiber" dimensions beyond space-time in the sense of gauge theory ${ }^{5}$ rather than the extra curled-up metric dimensions of KaluzaKlein theory. Finally, "transluminal" signals of the fourth kind occur in a shadow Riemannian metric of signature $+1++$ left over from the quantum gravity era of the early universe (to be discussed further in Sec. 2).

Signals of the third kind may be involved as the essential quantum mechanism of ordinary consciousness. Penrose, ${ }^{1}$ in a remarkable analysis, has led me to conclude that our common assumption of morally responsible "free will" demands controllable retroactive (backwards-in-time) action of the mind on matter by about two seconds in order to agree with EEG experiments. If we choose to hold on to the traditional notion of past cause and future effect, then we must be mere automatons with no free will. That is, consciousness of our actions is after the action. Causality then implies that consciousness is a passive epiphenomenon and not an active decision maker. This is not a view that I find to my liking.

\subsection{Stapp's First Argument Against Superluminal Quantum Signals of the Third Kind}

Consider the basic pair-correlation experiment ${ }^{6}$ involving spin correlations. A pair source $S$ emits pairs of spin-spin correlated particles 1 and 2 moving "back-to-back" in opposite directions to detectors A and B, respectively (see Fig. 1).

One kind of simple no-faster-than-light (FTL) signal argument ${ }^{7}$ is due to Stapp in a private communication. Stapp's analysis involves the use of projection operators. Suppose the pure pair quantum state is $|1,2\rangle$. Let the projection operators for the spin eigenvalues of each detector be $P_{\mathrm{A}(\mathrm{B}) \pm}$; then some of the nonlacal joint probabilities $p$ are

$$
p(\mathrm{~A}+\mid \mathrm{B}+)=\left\langle 1,2\left|P_{\mathrm{A}+} P_{\mathrm{B}+}\right| 1,2\right\rangle,
$$




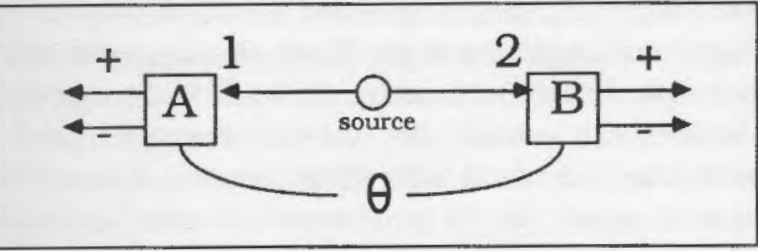

are 1. Basic pair Correlation experiment. The nonlocal parameter is the ade between the spin-sensitive detectors at the times the particles in the sure pair are detected. For this total experimental arrangement the local in probabilities are $1 / 2$ independent of the nonlocal parameter. Therefore, 1. antum signal of the third kind is not possible with this design. However, tere are other possibilities.

$$
p(\mathrm{~A}+\mid \mathrm{B}-)=\left\langle 1,2\left|P_{\mathrm{A}+} P_{\mathrm{B}-}\right| 1,2\right\rangle .
$$

One of these joint probabilities is what is actually measured in experiments correlating the outputs after the fact. Measurement of joint probabilities

the fact does show a FTL quantum spin-spin connection that violates a finn of the principle of causality expressed by Bell's inequality. However, is is generally accepted that this type of quantum causality violation called zcontrollable quantnm nonlocality" cannot be used for useful quantum zertuminal communication of the third kind, which, by definition, is in zel time."

i sufficient (but not necessary) condition for useful quantum superlumial communication of the third kind is that the local probability, for example $(+)$. changes as some controllable nonlocal parameter is changed. Imagz a sequence of twin pulses of photon pairs. The observer at receiver zatertor A would see the response of his detector in the + spin eigenvalue tarnel change in time caused by a change in the relative orientation of Ae rin transmitter detector at $\mathrm{B}$.

The pulse width must be small compared to the flight times from source erectors. The nonlocal parameter must not be changed during the time it it takes a single pulse to be detected. There must be enough photon as produced in each twin pulse to get a good signal-to-noise ratio in the iectors. The nonlocal parameter must be changed in a time that is short r.yared to the flight times. It must also be changed between the arrivals If suressive pulses.

I the flight times are equal, then the transmission and reception of if ration is essentially instantaneous in the rest frame of the apparatus.

1 te components of the apparatus are assumed to be at rest relative to acher. Now, let the flight time from pair source $S$ to transmitter $B$ be than the flight time to receiver $A$. The choice of nonlocal parameter at $\mathrm{B}$ can be delayed until after pulse 1 has been detected at receiver before its twin pulse 2 has been detected at transmitter B. The past spense at receiver A is then caused by a future cause at $B$. This would be asality violation in the strongest sense imaginable.

seth a phenomenon would place hitherto unsuspected limits on free will. ràng to Godel, any attempt to create a causal paradox in a universe ath trine travel would be doomed to failure: "... time travel is possible, but y person will ever manage to kill his past self. ... The a priori is greatly -lected. Logic is very powerful." Thus metaphorically there would have to z a new sort of nonmetrical transtemporal globally self-consistent type of iction at an arbitrary space-time distance in which controllably nonlocal quantum forces prevent a logical contradiction. Thome et al..$^{3}$ have discussed this possibility in the context of time travel to the past through traversable wormholes.

Retuming to Stapp's argument that retroactive communication is not possible at the quantum level,

$$
\begin{aligned}
p(\mathrm{~A}+) & =p(\mathrm{~A}+\mid \mathrm{B}+)+p(\mathrm{~A}+\mid \mathrm{B}-) \\
& =\left\langle 1,2\left|P_{\mathrm{A}+} P_{\mathrm{B}+}\right| 1,2\right\rangle+\left\langle 1,2\left|P_{\mathrm{A}+} P_{\mathrm{B}-}\right| 1,2\right\rangle \\
& =\left\langle 1,2\left|P_{\mathrm{A}+} P_{\mathrm{B}+}+P_{\mathrm{A}+} P_{\mathrm{B}-}\right| 1,2\right\rangle \\
& =\left\langle 1,2\left|P_{\mathrm{A}+}\left(P_{\mathrm{B}+}+P_{\mathrm{B}-}\right)\right| 1,2\right\rangle=\left\langle 1,2\left|P_{\mathrm{A}+}\right| 1,2\right\rangle
\end{aligned}
$$

because of the completeness of the spin eigenstates of transmitter $B$,

$$
P_{\mathrm{B}+}+P_{\mathrm{B}-}=1,
$$

and its associated conservation of local probability at B. Therefore, there is no dependence of the local receiver probability $\rho(A+)$ on any eigenvalue or nonlocal parameter that depends on B. Therefore, superluminal communication by quantum spin pair correlations is impossible. This completes Stapp's first argument against superluminal quantum signals of the third kind.

\subsection{The Flaw in Stapp's First Argument}

First of all, it must be admitted that Stapp's analysis does correctly describe the results of all actual experiments done so far with photon pairs that test Bell's inequality. The photon pair state for these experiments predicts that

$$
\begin{aligned}
& p(\mathrm{~A}+\mid \mathrm{B}+)=(1 / 2) \cos ^{2} \theta, \\
& p(\mathrm{~A}+\mathrm{B}-)=(1 / 2) \sin ^{2} \theta .
\end{aligned}
$$

Therefore,

$$
p(\mathrm{~A}+)=(1 / 2)\left(\cos ^{2} \theta+\sin ^{2} \theta\right)=1 / 2
$$

independent of the angle $\boldsymbol{\theta}$. So, indeed, there is no snperluminal commnnication. However, Stapp's equation

$$
p(\mathrm{~A}+)=p(\mathrm{~A}+\mid \mathrm{B}+)+p(\mathrm{~A}+\mid \mathrm{B}-)
$$

is an equation from classical probability theory that ignores the possible interference of quantum amplitudes. This is the flaw in Stapp's analysis. It does not apply to total experimental arrangements in which at least one of the photons in the same pair interferes with itself. For the experiments actually done, however, it is the correct equation. This follows from Feynman's quantnm rules.

\subsection{Feynman's Quantum Rules}

(1) Square the amplitudes before adding for distinguishable alternatives.

(2) Add the amplitudes before squaring for indistinguishable altematives.

In the basic pair-correlation experiment there are two photon counters at each detector, which is basically equivalent to a doubly refracting calcite crystal. Therefore, all the nonlocal alternatives, $(\mathrm{A}+\mathrm{B}+),(\mathrm{A}+, \mathrm{B}-),(\mathrm{A}-$, 


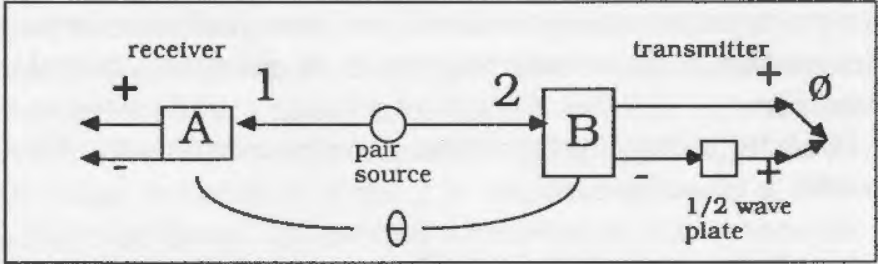

Figure 2. Pair correlation interference experiment. The transmitter photon 2 interferes with itself and causes a controllahle shift in the polarization of its far-away twin receiver photon 1 that depends upon the angle between the calcite crystals at the moments of detection, and upon the phase shift in the transmitter interferometer.

$\mathrm{B}+)$, and $(\mathrm{A}-, \mathrm{B}-)$, are distinguishable. Hence Stapp's equation is justified for this class of total experimental arrangements. But is it justified for all possible experiments? No, it is not (see Fig. 2).

There is now a new parameter $\phi$ in the experiment, the translational phase difference of the two indistinguishable alternatives for transmitter photon 2. Experiments with neutron interferometers show that it is possible to construct an $x$ spin state from the coherent interference of two orthogonal $+y$ and $-y$ spin states. On the other hand, with photons we know that a double-slit experiment with orthogonal linear polarizers at each slit destroys the interference fringes. That does not, in principle, mean that coherent interference is not happening in the photon case. Indeed, the interference shows up in the change of polarization state at different points of the screen. In some places there will be left-handed circular, in other places right-handed circular, etc. This only means that a more sophisticated kind of measurement would need to be done at the screen of the donble slit to detect coherence from orthogonally polarized paths.

The experiment we are interested in does not depend upon whether there are interference fringes for photon 2 at the transmitter $\mathrm{B}$. What we really care about is whether the degree of linear polarization of a stream of photons 1 at the receiver $A$ can be controlled at a distance from $B$. This nonlocal control would be achieved by keeping the orientation of A fixed but rotating $\mathrm{B}$ relative to $\mathrm{A}$ in time. I have placed a half-wave plate in one arm of the interferometer.

Interference fringes will he seen if the half-wave plate is in place for ordinary uncorrelated light. In fact, however, local interference fringes will not appear at the transmitter even if the half-wave plate is in place. This is because there are two mutually out-of-phase nonlocal interferograms corresponding to the distinguishable alternatives of receiver photon 1 at $\mathrm{A}$ These two nonlocal interferograms ${ }^{(4)}$ could be resolved after the fact by coincidence analysis. Their existence might be exploited for an untappable, unbreakable quantum cryptographic military intelligence application. However, this cryptographic application has nothing to do with the present problem of superluminal signaling. I mention it in passing for its intrinsic interest.

Feynman's rules for the present interference pair correlation experiment imply that we must add the amplitudes $\langle 1,2 \mid \mathrm{A}+, \mathrm{B}+\rangle$ and $\epsilon(\phi)\langle 1,2| \mathrm{A}+$, $\mathrm{B}-\rangle$ before squaring. Similarly, we must add the amplitudes $\langle 1,2 \mid \mathrm{A}-, \mathrm{B}+\rangle$ and $\epsilon(\phi)\langle 1,2 \mid \mathrm{A}-, \mathrm{B}-\rangle$ before sqnaring. What do we get after we square? What we get is a pair of nonlocal joint probabilities that photon 1 takes a particular channel at A and that its twin photon 2 is detected at B with a definite translational phase difference $\phi$.
Are we justified in ignoring the space-time dependence of the pair amplitudes? Experiment suggests that we are. There is no evidence of a weakening in the strength of $(A+B+)$ coincidence correlation as the separation between the detectors is increased. This $(A+B+)$ correlation is $1 / 2 \cos ^{2} \theta$ in the unattainable ideal case of $100 \%$ efficient detection. More experiments are needed. It appears that the spin-correlated pair wave function simply multiplies two independently moving uncorrelated space-time wave packets for each photon. This would be consistent with Ne'eman's already quoted fiber bundle picture": "The two $\gamma$ rays should have their spin polarizations adding up to zero (and to negative total intrinsic parity) when observed whatever the $\mathrm{AB}$ distance. ... in a fiber bundle geometry, the manifold is constrained so as to preserve parallelism whatever the magnitude of the base space interval." That is, the relevant quantum connection in this case is entirely in spin-fiber space beyond space-time.

The problem for superluminal signaling is how to correctly take the sum of distinguishable altematives over all possible places where the transmitter photon 2 can be absorbed at B. This is the basis for Stapp's second argument against superluminal communication of the third kind. Stapp's motivation is the true fact that coherent interference redistributes the conserved local probability density. If one integrates the interference cross terms over all possible places where the single transmitting photon might be absorbed, then the cross terms integrate to zero. What Stapp fails to realize is that not all these places arerelevant in computing the controllable nonlocal quantum action-at-a-distance on the far-away twin receiver photon. If the dependence of the difference in the local receiver probabilities $p(\mathrm{~A}+)-p(\mathrm{~A}-)$ on $\theta$ (i.e., the superluminal signal of the third kind) survives the sum over $\phi$, then we are in the superluminal communication business. If that tums out to be the case, we can manipulate the degree of partial polarization of receiver light at a distance from the future in a delayed-choice mode of operation of the device.

The message would then be contained in the encoding modulating function $\theta\left(t_{2}\right)$, which maps to the decoding modulating function $\boldsymbol{\theta}\left(t_{1}+\left[L_{2}-L_{1}\right] / c\right)$, where

$$
t_{1}=t_{2}-\left(L_{2}-L_{1}\right) / c \text {, }
$$

and $L_{1}$ and $L_{2}$ are the spatial distances of the detectors $\mathrm{A}$ and $\mathrm{B}$ from the source $S$, respectively. So, if $L_{2}>L_{1}$, the cause at time $T_{2}$ is in the future of the effect at time $t_{1}$. That is, the degree of polarization of the receiver light at A at time $t_{1}$ is the function $F$ of time $t_{2}$ :

$$
p\left(\mathrm{~A}+, t_{1}\right)-p\left(A-, t_{1}\right)=F\left\{\theta\left(t_{1}+\left[L_{2}-L_{1}\right] / c\right)\right\} .
$$

Analyzers A, B and source S are all relatively at rest along the line of flight of the photon pair. One way of encoding the message at B is by a variable rotation $d \theta / d t$ of the entire B assembly about the axis of the line of flight. However, I will calculate $F$ explicitly for the gedanken experiment given below, and we will see that there is an easier way to do it using the $\phi$ dependence.

\subsection{The Photon Pair State}

Start with the standard photon pair state actually used in and confirmed by experiment. The photons are emitted back-to-back in opposite directions with total angular momentum zero. Consider a double quantum jump of an atomic electron for which the emitted photon pair state is even under parity mirror imaging. Therefore, for linear polarizations the directions of 
polarization are parallel. The directions of rotation for circular polarizations are opposite (for one observer looking from a fixed point of view). The helicities (projections of spin along momentum) are the same. Odd parity pair states have perpendicular linear polarizations. All this is independent of how far apart in space or time the detections of each photon in the same pair are.

The Einstein-Podolsky-Rosen (EPR) paradox consists in precisely this fact together with the realization that we do not have to decide until the last instants prior to the detections whether to locally measure circular polarization or, alternatively, linear polarization in any direction (transverse to the line of flight). No matter what we arbitrarily decide, the far-away photons will always show parallel linear polarizations or opposite circular polarizations (equal helicities). Each photon "knows" how the other photon is being measured.

Suppose we have only one photon passing two polarizers. The first polarizer prepares the photon in a given spin state. The second polarizer analyzes the prepared state. This is easy to understand locally. A similar thing happens nonlocally for our photon pair. Photon 1 passes a polarizer A. Not only does $A$ locally prepare photon 1 in a definite state with probability $1 / 2$, it also nonlocally prepares its distant twin photon 2 in the same state as the one sctually allegedly randomly chosen for photon 1 . Therefore, when photon 2 encounters its polarizer at B, it will simply be analyzed in the state in - thich A prepared it at a distance. Analyzer A's preparation of photon 2 can be in the future of photon 2's encounter with analyzer B. The situation is totally symmetrical. We can say that B prepares photon 1 at a distance, which is then analyzed by A, etc.

Thus, start from the (R, L) circular polarization frame of reference in spin fiber space beyond space-time for a single observer looking from a fixed point of observation along the line of flight.

Note that $\psi_{1}\left(x_{1}\right) \psi_{2}\left(x_{2}\right)$ are the two uncorrelated wave packets of each photon moving in opposite directions in space. Their function is simply to deliver the photon energies to the detectors. They do not carry the message in modulated energy flows the way that they do for signals of the first and second kind. Their role in the FTL spin fiber signal of the third kind is recessary but secondary:

$$
|1,2\rangle=(1 / \sqrt{ } 2)\left[\left|L_{1}\right\rangle\left|R_{2}\right\rangle+\left|R_{1}\right\rangle\left|L_{2}\right\rangle\right] \psi_{1}\left(x_{1}\right) \psi_{2}\left(x_{2}\right) .
$$

Photons are bosons, and, therefore, the wave functions for several identical fhotons must be totally symmetric in all the quantum numbers. However, in the present case the two photons in the same pair have nonoverlapping szre packets with different peak frequencies and different line widths and, terefore, are not identical. Thus the photon pair function (11), though sfmmetrical in spin space, need not be so in physical space.

Make a spin fiber frame shift to a particular linear polarization basis (V, H) and substitute

$$
\begin{gathered}
|\mathrm{L}\rangle=(1 / \sqrt{ } 2)[|\mathbf{V}\rangle+i|\mathbf{H}\rangle], \\
|\mathrm{R}\rangle=(1 / \sqrt{ } 2)[|\mathbf{V}\rangle-i|\mathbf{H}\rangle], \\
1,2\rangle=(1 / 2 \sqrt{ } 2)\left[\left\{\left|\mathbf{V}_{1}\right\rangle+i\left|\mathbf{H}_{1}\right\rangle\right\}\left\{\left|\mathbf{V}_{2}\right\rangle-i\left|\mathbf{H}_{2}\right\rangle\right\}\right. \\
\left.+\left\{\left|\mathbf{V}_{1}\right\rangle-i\left|\mathbf{H}_{1}\right\rangle\right\}\left\{\left|\mathbf{V}_{2}\right\rangle+i\left|\mathbf{H}_{2}\right\rangle\right\}\right] \Psi_{1}\left(x_{1}\right) \Psi_{2}\left(x_{2}\right),
\end{gathered}
$$

$$
|1,2\rangle=(1 / \sqrt{ } 2)\left[\left|\mathbf{V}_{1}\right\rangle\left|\mathbf{V}_{2}\right\rangle+\left|\mathbf{H}_{1}\right\rangle\left|\mathbf{H}_{2}\right\rangle\right] \Psi_{1}\left(x_{1}\right) \psi_{2}\left(x_{2}\right)
$$

Now, make two arbitrary independent local frame shifts from the totally arbitrary common $(\mathbf{V}, \mathbf{H})$ basis of both photons to the bases $\left(V_{1}, H_{1}\right)$ and $\left(V_{2}^{\prime}, H_{2}^{\prime}\right)$. These bases correspond to the actual orientations $\theta_{\mathrm{A}}\left(t_{1}\right)$ and $\theta_{\mathrm{B}}\left(t_{2}\right)$ of the calcite crystals at A at time $t_{1}$ and B at time $t_{2}$ where, as defined above,

$$
t_{1}=t_{2}-\left(L_{2}-L_{1}\right) / c
$$

Substitute

$$
\begin{gathered}
\left|\mathbf{V}_{1}\right\rangle=\cos \theta_{\mathrm{A}}\left(t_{1}\right)\left|V_{1}\right\rangle+\sin \theta_{\mathrm{A}}\left(t_{1}\right)\left|H_{1}\right\rangle, \\
\left|\mathbf{H}_{1}\right\rangle=-\sin \theta_{\mathrm{A}}\left(t_{1}\right)\left|V_{1}\right\rangle+\cos \theta_{\mathrm{A}}\left(t_{1}\right)\left|H_{1}\right\rangle, \\
\left|\mathbf{V}_{2}\right\rangle=\cos \theta_{\mathrm{B}}\left(t_{2}\right)\left|V_{2}^{\prime}\right\rangle+\sin \theta_{\mathrm{B}}\left(t_{2}\right)\left|H_{2}^{\prime}\right\rangle, \\
\left|\mathbf{H}_{2}\right\rangle=-\sin \theta_{\mathrm{B}}\left(t_{2}\right)\left|V_{2}^{\prime}\right\rangle+\cos \theta_{\mathrm{B}}\left(t_{2}\right)\left|H_{2}^{\prime}\right\rangle .
\end{gathered}
$$

Define the nonlocal modulation parameter

$$
\boldsymbol{\theta}(\tau-2) \equiv \boldsymbol{\theta}_{\mathrm{A}}\left(t_{1}\right)-\boldsymbol{\theta}_{\mathrm{B}}\left(t_{2}\right),
$$

and let us agree to keep the receiver orientation $\theta_{\mathrm{A}}\left(t_{1}\right)$ fixed in time in what follows when we get to FIL communication. Familiar trigonometric identities give us the useful form of the photon pair state $|1,2\rangle$ in terms of the actual orientations of the birefringent calcite crystals at the detectors $A$ and $B$. That is,

$$
\begin{aligned}
|1,2\rangle= & (1 / \sqrt{ } 2)\left[\cos \theta(1-2)\left\{\left|V_{1}\right\rangle\left|V_{2}^{\prime}\right\rangle+\left|H_{1}\right\rangle\left|H_{2}^{\prime}\right\rangle\right\}\right. \\
& \left.+\sin \theta(1-2)\left\{\left|V_{1}\right\rangle\left|H_{2}^{\prime}\right\rangle-\left|H_{1}\right\rangle\left|V_{2}^{\prime}\right\rangle\right\}\right] \Psi_{1}\left(x_{1}\right) \Psi_{2}\left(x_{2}\right) .
\end{aligned}
$$

There is no need to worry about the relativistic collapse of the wave function. ${ }^{(5)}$ If one wishes to imagine that the wave function collapses instantly, then there is no problem with relativity, because we are explicitly assuming that causality is wrong. Future causes are allowed. But even more importantly, we can do away with the notion of instantaneous collapse. Operationally we have two measurements made at an arbitrary space-time separation from each other. As Wheeler says, "no quantum phenomenon is a phenomenon until it is an observed phenomenon." Thus what we are talking about is not even defined until both detections are made. There is no independent way to detect the collapsing wave function between the two detections. It is a pseudoproblem. What we have, here, is a nonlocal, nonmetrical global quantum phenomenon, which is basically happening beyond space-time. It is premetrical at a deeper level, that is, a bigger Klein-Erlanger group $^{8}$ than the Lorentz group metrical geometry of relativity.

\subsection{The Gedanken Experiment}

In Fig. 3 a half-wave plate converts $\left|H_{2}^{\prime}\right\rangle$ to $\left|V_{2}^{\prime}\right\rangle$ at the transmitter. A half-silvered mirror is at the transmitter crossing point $\mathcal{B}$. Those transmitter photons 2 whose twin photons 1 are detected in the $V_{1}$ channel will form a nonlocal interferogram on the transmitter screen; similarly for those transmitter photons 2 whose twin photons 1 are detected in the $H_{1}$ channel. Fully reflecting mirrors $(\mathcal{A}, \mathcal{C}, \mathcal{D})$ bring these two mutually out-of-phase nonlocal interferograms to a small region $\mathcal{E}$ on the transmitter screen. 


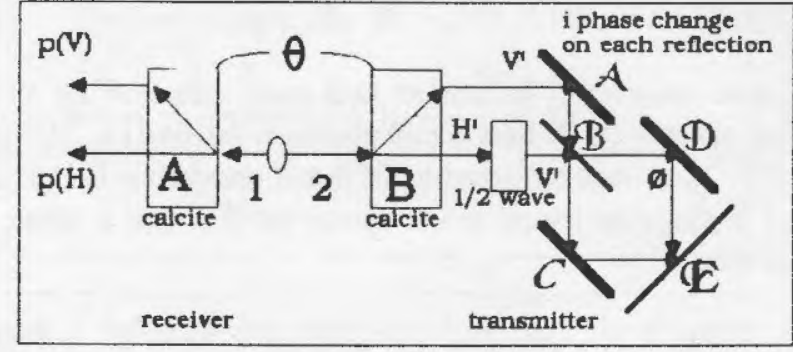

Figure 3. The gedanken experiment.

Thus no fringes will be visible at the transmitter screen for pair-correlated light. Fringes would be visible if the source emitted uncorrelated photons 2 with the same spectral line shape and spatial coherence of photon 2 in the pair-correlated case. As already mentioned ${ }^{(4)}$ above, the two nonlocal interferograms can be disentangled from each other by coincidence measurements for one photon pair in the system at a time.

\subsection{The Feynman Photon Pair Probability Amplitudes}

The relative phase difference between the interferograms acquired between the crossing point $\mathcal{B}$ and $\mathcal{E}$ on the screen is $\phi(2)$. There is a phase shift of $i$ for each reflection, and an extra factor of $1 / \sqrt{ } 2$ from the half-silvered mirror at $\mathcal{B}$. The Feynman path amplitudes are

$$
\begin{gathered}
\mathrm{I}=\left\langle 1,2 \mid V_{1}, V_{2}^{\prime}, \mathcal{A}, \mathcal{B}, \mathcal{D}, \mathcal{E}\right\rangle=(1 / 2) \cos \theta(1-2) i^{3} e^{i \phi(2)}, \\
\mathrm{II}=\left\langle 1,2 \mid V_{1}, H_{2}^{\prime}, \mathcal{B}, \mathcal{D}, \mathcal{E}\right\rangle=(1 / 2) \sin \theta(1-2) i e^{i \phi(2)}, \\
\mathrm{III}=\left\langle 1,2 \mid V_{1}, V_{2}^{\prime}, \mathcal{A}, \mathcal{B}, \mathcal{C}, \mathcal{E}\right\rangle=(1 / 2) \cos \theta(1-2) i^{2}, \\
\mathrm{IV}=\left\langle 1,2 \mid V_{1}, H_{2}^{\prime}, \mathcal{B}, \mathcal{C}, \mathcal{E}\right\rangle=(1 / 2) \sin \theta(1-2) i^{2}, \\
\mathrm{~V}=\left\langle 1,2 \mid H_{1}, V_{2}^{\prime}, \mathcal{A}, \mathcal{B}, \mathcal{D}, \mathcal{E}\right\rangle=-(1 / 2) \sin \theta(1-2) i^{3} e^{i \phi(2)}, \\
\mathrm{VI}=\left\langle 1,2 \mid H_{1}, H_{2}^{\prime}, \mathcal{B}, \mathcal{D}, \mathcal{E}\right\rangle=(1 / 2) \cos \theta(1-2) i^{i \phi(2)}, \\
\mathrm{VII}=\left\langle 1,2 \mid H_{1}, V_{2}^{\prime}, \mathcal{A}, \mathcal{B}, \mathcal{C}, \mathcal{E}\right\rangle=-(1 / 2) \sin \theta(1-2) i^{2}, \\
\mathrm{VIII}=\left\langle 1,2 \mid H_{1}, H_{2}^{\prime}, \mathcal{B}, \mathcal{C}, \mathcal{E}\right\rangle=(1 / 2) \cos \theta(1-2) i^{2}
\end{gathered}
$$

The Feynman rules of standard quantum mechanics tell us to add the amplitudes before squaring for indistinguishable alternatives, and to square the amplitudes before adding for distinguishable alternatives. Clearly, with the above total experimental arrangement, the eight alternatives form two distinct sets of indistinguishable alternatives (nonlocal interferograms), that is, $\{\mathrm{I}, \mathrm{II}, \mathrm{III}, \mathrm{IV}\}$ and $\{\mathrm{V}, \mathrm{VI}, \mathrm{VII}, \mathrm{VIII}\}$. The first set corresponds to a measurement of receiver photon 1 in the $\left|V_{1}\right\rangle$ state. The second set corresponds to the receiver photon in the $\left|H_{1}\right\rangle$ state. Therefore,

$$
\begin{aligned}
p_{+}^{\prime}\left(V_{1}, \theta(1-2), \phi(2)\right) & =|I+I I+I I I+I V|^{2} \\
= & (1 / 2)[1-\cos 2 \theta(1-2) \sin \phi(2)], \\
p_{-}^{\prime}\left(H_{1}, \theta(1-2), \phi(2)\right) & =|V+V I+V I I+V I I I|^{2} \\
= & (1 / 2)[1+\cos 2 \theta(1-2) \sin \phi(2)] .
\end{aligned}
$$

These are the nonlocal joint probabilities for coincidence measurements in which the receiver photon 1 is observed to have polarization eigenvalue $V_{1}\left(H_{1}\right)$, and its twin transmitter photon 2 is observed to land on the screen with translational phase difference $\phi(2)$ when the calcite crystals are misaligned by the nonlocal angle $\theta(1-2)$ connecting the two arbitrarily separated detection events.

\subsection{The Transmitter Phase Noise}

The superluminal signal at the receiver is derivable from the difference between these squared pair amplitudes when properly summed over all relexant values of the phase difference $\phi(2)$. This is the crux of the debate. The controversy is over how to perform this sum. For this particular experimental arrangement the overlap area $\mathcal{E}$ is small. The $L$ is the variable path difference between $\mathcal{B D E}$ and $\mathcal{B C E}$, that is,

$$
L=(\mathcal{B D E}-\mathcal{B C E}) .
$$

For uniform index of refraction $n$

$$
\begin{gathered}
\phi(2)=2 \pi L n / \lambda_{2}, \\
L<c / n \delta \mathrm{v}_{2},
\end{gathered}
$$

that is, the path difference $L$ must be smaller than the coherence length of the transmitter photon wave packet $g\left(x_{2}\right)$. The fluctuation in $\phi(2)$ is $\delta \phi$. It is due to variations in three variables, $L, n$, and $\lambda_{2}$, that is,

$$
\delta \phi=2 \pi\left[(\delta L) n / \lambda_{2}+L\left(\delta n / \lambda_{2}\right)+L n \delta\left(1 / \lambda_{2}\right)\right]
$$

Therefore, if we choose a particular $L_{0}$ obeying the coberence condition Eq. (35), we have some mean $\phi_{0}$. The relevant range of $\phi$ integration is then $\phi_{0} \pm \delta \phi$.

\subsection{The Error in Stapp's Second Argument Against Superluminal Signals}

Henry Pierce Stapp of the Lawrence Berkeley Laboratory, in private communication, has objected that a superluminal interference signal violates conservation of local probability $p(2)$ for the transmitter photon 2 . This second objection is that after properly pefforming the sum over all places where the transmitter photon 2 might be absorbed on the screen, the dependence of the local receiver probabilities $p(V)$ and $p(H)$ (see Fig. 3) on the misalignment angle $\theta(1-2)$ will cancel. This would destroy the nonlocal quantum signal. On the contrary, I argue that while all places where the transmitter photon 2 can be absorbed do contribute to conserving the local probability $p(2)$, many of those places do not contribute to the nonlocal signal $p(V)-p(H)$ in the far-away spacelike separated receiver region. Only a subset of those places where photon 2 might land contribute to the detection probability of its twin photon 1 . That is, the waves arriving at $\mathcal{E}$ at the transmitter must coberently interfere in order to produce the signal at the far-away receiver. Like the quantum signal in the Josephsou effect, the superluminal signal is essentially a macroscopic quantum interference phenomenon using photon pairs, rather than electron pairs.

The second objection, however, was in the context of a double-slit arrangement, not the arrangement of Fig. 3 in which the phase variation $\phi$ is more tightly controlled. Thus $\delta \phi$ is understood to mean the uncertainty in phase coming from that coherent subset of places where the transmitter photon might be absorbed in which the waves from paths $\mathcal{B D E}$ and $B C E$ are still mutually coberent. If they are not coherent, then they have 
no controllable nonlocal effect at the spacelike separated receiver, although they certainly figure in conserving local probability $p(2)$ at the transmitter screen.

\subsection{Normalization of the Transmitter Photon Probabilities}

First, normalize the transmitter photon probability density in area $\mathcal{E}$ of coherent interference of the waves from the altemate paths in the interterometer. Take the point of view of the local observer at the transmitter screen. If a transmitter photon 2 arrives, it could have its spacelike separated win receiver photon arrive in either the $V_{1}$ channel or the $H_{1}$ channel. These are distinguisbable, noninterfering alternatives, because there is a separate photon counter in both the $V_{1}$ and $H_{1}$ channels. Therefore, the un-normalized transmitter photon probability density at the screen area $\mathcal{E}$ is simply the sum of $p^{\prime}\left(V_{1}, \theta(1-2), \theta(2)\right)$ with $p^{\prime}\left(H_{1}, \theta\right.$, $(1-2), \phi(2)))$ from Eqs. (31) and (32). This sum is 1 and is the reason aty no local interference fringes are seen with pair-correlated light. On the ather hand, the normalized probability densities $p\left(V_{1}, \theta(1-2), \phi(2)\right)$ and $p\left(H_{1}, \theta(1-2), \phi(2)\right)$ conserve local probability only for the subset of coherent photon pairs whose transmitter photon 2 interferes with itself. Only that coberent subensemble of photon pairs contribute to the nonlocal signal of the third kind $p(V)-p(H)$. Photon pairs whose transmitter photon 2 does not interfere with itself belong to a different incoherent subensemble, -hich does not contribute to the nonlocal signal. These incoherent pairs are irelevant to the computation of the sum over all places where the transmitex photon 2 might land within the coherent region of $\mathcal{E}$. Therefore [call $\theta(1-2)$ simply $\theta$, and $\phi(2)$ simply $\phi]$,

$$
\int_{\phi_{0}-\delta \phi}^{\phi_{0}+\delta \phi} d \phi[p(V, \theta, \phi)+p(H, \theta, \phi)]=1
$$

Therefore,

$$
p(H(V), \theta, \phi)=[1 \pm \cos 2 \theta \sin \phi] / 4 \delta \phi .
$$

The sum over transmitter phases that survives at the spacelike separated seceiver is

$$
\begin{aligned}
\langle\sin \phi\rangle & =\int_{\phi_{0}-\delta \phi}^{\phi_{0}+\delta \phi} d \phi \sin \phi[p(V, \theta, \phi)+p(H, \theta, \phi)] \\
& =\frac{\int_{\phi_{0}-\delta \phi}^{\phi_{0}+\delta \phi} d \phi \sin \phi}{2 \delta \phi}=\sin \phi_{0} \frac{\sin \delta \phi}{\delta \phi}=\sin \phi_{0} \sin c \delta \phi .
\end{aligned}
$$

Clearly, the average of $\sin \phi$ over the transmitter photon normalized r bability distribution for $\phi$ in the coberent subregion of $\mathcal{E}$ on the screen s rot generally zero. In fact, it depends on the average transmitter interferreter phase difference $\phi_{0}$ and its actual uncertainty $\delta \phi$ due to fluctuations in the relevant experimental parameters.

I have assumed that the altematives for different $\phi$ are noninterfering, wa they correspond to an irreversible absorption of a photon completing te measurement or "making a record." That is, one can consider the screen D be a retinalike array of photon detectors.

\subsection{The Superluminal Signal at the Receiver}

Thus the superluminal receiver signal $S$ is the degree of partial linear priarization of the receiver photon, which is the difference of the local roabilities $p(H, \theta)$ and $p(V, \theta)$, that is,

$$
\begin{aligned}
& p(H(V), \theta)=\int_{\phi_{0}-\delta \phi}^{\phi_{0}+\delta \phi} d \phi p(H(V), \theta, \phi) \\
&=\frac{1}{2}[1 \pm \cos 2 \theta\langle\sin \phi\rangle] \\
&=\frac{1}{2}\left[1 \pm \cos 2 \theta \sin \phi_{0} \sin c \delta \phi\right], \\
& S=p(H, \theta)-p(V, \theta)=\cos 2 \theta \sin \phi_{0} \sin c \delta \phi .
\end{aligned}
$$

Equation (41) says that the degree of linear polarization of the receiver photon can be controlled across spacelike (even timelike) intervals at a distance, indeed, even backwards in time. Note that the signal $S$ can be modulated by either varying $\theta$ or $\phi_{0}$. As a practical matter it would be much easier to modulate $S$ by changing $\phi_{0}$ with fixed $\theta$. Notice, also, that local probability is clearly conserved on both sides of the apparatus, even though there is a nonlocal superluminal signal.

\subsection{Competing Designs for FTL Communicators}

The first published "FLASH" design is by Herbert. ${ }^{(6)}$ It is generally believed to be unworkable, because the proposed "photon-cloning" laser amplifying decoding mechanism appears to violate the superposition principle of quantum mechanics. However, the basic idea that Herbert had is interesting and can, perhaps, be made to work.

Herbert's idea is totally different from the one proposed in this paper. The reason Herbert's idea may eventually be made to work is the recent progress in chaos. Thus it may be that sequences of individual quantum events (IQE's) that directly feel the quantum connection are not really random, but contain universal hidden fractal orders whose locally observable signatures depend in a controllable way upon distant actions. This general approach is also alluded to as "symmetric-time," which is at a deeper level than irreversible "directed-time". (7) Thus "All combinatorial choices made under symmetric causality are consistent with the quantum probability interpretation of directed causality. [p. 141] ... A complex dual process may be consistent with quantum mechanics simply by virtue of its pseudorandomness, despite having a unique selection property over space-time. [p. 143]" There are practically no experiments on this very fundamental problem.

Herbert accepts the standard notion that it is impossible in principle to shift quantum probabilities at a distance. I am denying that the sequence of quantum events at one end of the photon pair experiment need always be uncontrollably random for all possible total expenimental arrangements. It is further asserted that the departure from randomness of the sequence of quantum events can be controlled from an arbitrary distance across spacetime without the local action of a Hamiltonian. Quantum probabilities are locally shifted all the time by energy flows. What is novel is the shift of quantum probabilities by pure information flows in fiber space without corresponding energy flows in base space-time. Herbert believes that the quantum connection only acts controllably at the level of individual quantum events (IQE's) and is washed out in the statistical average over many events. On the contrary, I am asserting that the quantnm connection can be used to controllably shift the averages from a distance and that one does not have to go beyond standard quantum mechanics to achieve all this. One simply has to use Feynman's rules and a little bit of physical intuition.

Svozil, ${ }^{(8)}$ of the Institute for Theoretical Physics in Vienna, has a variation on FLASH that he calls SLASH (second-laser-amplified superluminal hookup). Herbert's FLASH had the laser amplifier at the receiver. Svozil's laser amplifier 


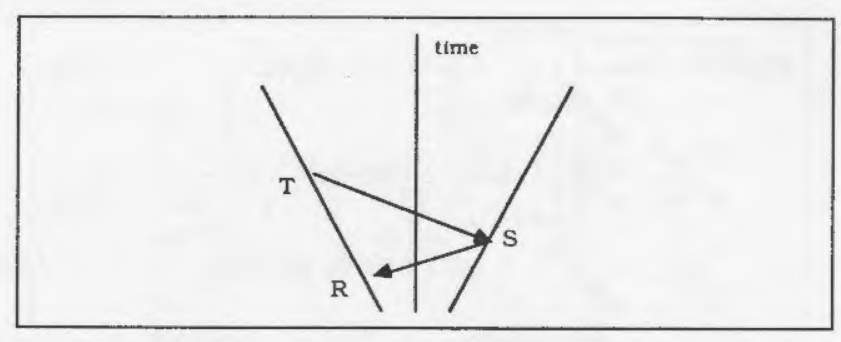

Figure 4. Autocidal causal anomaly using tachyon signals. If $\mathrm{R}$ receives YES, then $\mathrm{T}$ transmits NO to $\mathrm{S}$. If $\mathrm{R}$ receives NO, then T transmits YES to $\mathrm{S}$. If $S$ receives YES, $S$ transmits YES to $R$. If $S$ receives NO, $S$ transmits NO to $R$. This results in a self-referential paradox like the ones considered by Bertrand Russell and Alfred North Whitehead in Principia Mathematica and which finally led to Godel's incompleteness theorem.

is at the transmitter. While Svozil does not use interference, his gedanken experiment is more like the one in this paper than like FLASH. Syozil is not working at the hidden-variable level, but is attempting to use standard quantum mechanics to shift the receiver quantum probabilities at a distance. Syozil has not yet published his idea so that it would not be appropriate to discuss its details here.

\subsection{Autocidal Causal Anomaly}

(The following section was written in an earlier draft received by Pbysics Essays before I knew of the recent work on the global self-consistency of events on the closed timelike curves (CTC) ${ }^{3}$ generated by traversable wormholes built with matter violating the weak energy condition. Note that I give essentially the same sort of criterion in terms of Feynman histories that Thorne et al. now advocate.)

Suppose we use signals of the second kind (see Fig. 4). This is not essential; the same apparent paradox arises with signals of the third kind in the gedanken experiment above. The causal order of earlier-later between spacelike separated events T and S outside each other's light cones is subjective, changing with the frame of reference of the observer. The same is true of $S$ and $R$. But the net result is a retroactive signal backwards in time from $\mathrm{T}$ to $\mathrm{R}$ which is objectively separated by a frame-invariant time-like interval. Figure 4 follows Penrose's ${ }^{1}$ Fig. 5.32, p. 213.

Suppose the equipment is $100 \%$ reliable. This is the same as supposing that we have absolute free will. Then, indeed, if there are not parallel universes, there is a paradox. But both of these assumptions can be wrong. first, suppose that there are no parallel universes. There is only one unique universe. In that case the nonlocal quantum force can act at a 4-D distance and cause an error in order to keep the loop in time logically self-consistent. This is the position taken both by Hoyle ${ }^{1}$ and by the late Godel. ${ }^{9}$

In this extreme case the equipment will fail $100 \%$ of the time somewbere along the line. That is, any attempt to create a time-travel paradox will induce malfunctions in the equipment. For example, $\mathrm{R}$ can receive NO, T can transmit YES; if that happens, $S$ will malfunction. Or, another alternative, $\mathrm{R}$ receives NO, an error occurs so that T transmits $\mathrm{NO}$ and $\mathrm{S}$ does not make an error.

We can use Feynman's path quantum mechanics to describe all this. Every possible history has a quantum amplitude. The Feynman amplitudes for those bistories that are self-contradictory simply vanish. This is no more peculiar than, for example, the Pauli exclusion principle, which selects

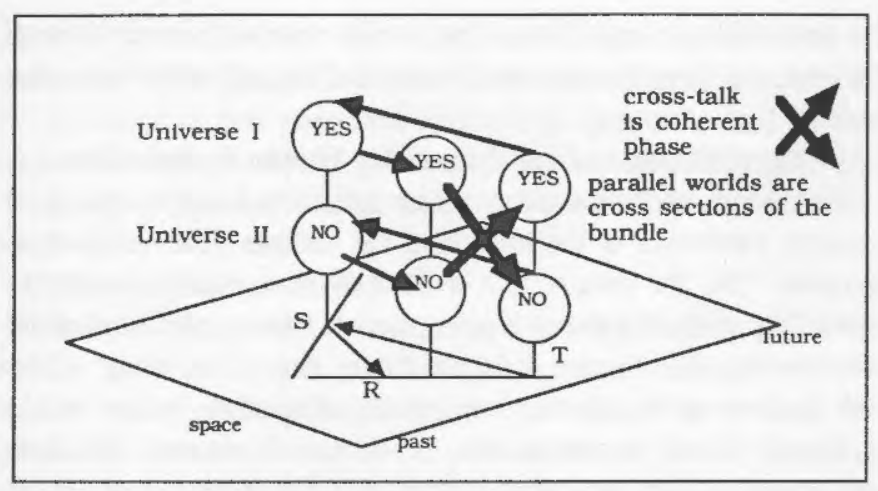

Figure 5. Doubly connected loop in time has topology of the double-sheeted Riemann surface linking parallel universes (sheets), and suppresses the autocidal causal anomaly.

out only antisymmetric states for systems of identical fermions. Quantum phenomena are inherently nonlocal, and nonlocality ensures self-consistent loops in time. Let us also remember that there are two important cases of classical nonlocality, that is, precognitive charged particle motions avoiding runaway solutions in Maxwell theory, and the nonlocality of gravitational energy in general relatiyity. We simply have to get used to nonlocality.

Let us suppose that there are parallel universes. This is the only interpretation that seems reasonable in quantum cosmology where we need a "wave function of the universe." In the standard parallel universe interpretation the copies of the same mind in different parallel universes cannot cross-talk to each other. Penrose has come to doubt this restriction ${ }^{1}$ and so do I. It is easy to see, using fiber bundle inspired pictures, that the causal anomaly induces cross talk or a doubly connected quantum loop in time. The double loop resembles the double-valuedness of the spinor under $2 \pi$ rotations of the frame of reference.

Figure 5 shows only one of several ways to connect the parallel universes into a self-consistent doubly-connected loop in time. The same topological idea is used in the theory of Riemann surfaces of functions of a complex variable (i.e., $\sqrt{ } z$ ). There, the point is to convert the multivalued function into a single-valued function. The demand for single-valuedness is analogous to our demand for nonlocal or global self-consistency.

The two points of view, that is, a single universe with malfunctioning simple loops in time and parallel universes with doubly connected loops in time, are actually equivalent. In Fig. 5 the mind clones, thinking they are only in one universe I (or II), will perceive that S functioned without error, but that there was an error in the RT link. For example, the mind clone trapped in universe II will perceive the following sequence of events: $R$ receives NO, but there is an error when T transmits NO to $S$, which correctly sends NO to R. So, we can have simple loops in time with errors, like multivalued complex functions, or we can have doubly connected loops in time linking normally unlinked parallel universes with no errors. Take your pick!

\section{RELATIVITY WITHOUT CAUSALITY}

You know, I have recently lost confidence in the principle of no action at a distance ...

Einstein to Ernst Straus, Some Strangeness in the Proportion ${ }^{(9)}$ 
Physics today only recognizes the existence of what I call the signal of the first kind." This is a one-way signal in the direction of time's anow that conveys useful messages from a past state of a transmitteremitter to a future state of a receiver-absorber. Messages are communicated by the controlled modulation of four-vector energy-momentum along either timelike or lightlike world lines. The energy-momentum flow for real particles is everywhere either inside or on the local light cone, even in curved spacetime. Therefore, the direction of causation from past to future is a frameinvariant distinction. It is interesting to note, however, that virtual photons are not confined to the light cone. Indeed, in the exchange of a single virtual photon between two charges, four-momentum conservation requires that the virtual photon is spacelike outside the light cone. Thus the near field Coulomb force really is superluminal - although this particular effect cannot be used to transmit useful messages.

It is the principle of causality that effects are always after causes in all frames of reference that places the speed of light barrier in Einstein's classical theory of special relativity. ${ }^{10}$ However, the principle of causality is to relativity s Euclid's fifth axiom of a unique parallel to a line through a point not on the line is to geometry. Thus causality is an additional postulate to the smmetry group of special relativity. The classic tests of special relativity of trne dilation and mass-energy equivalence do not depend upon the causality rostulate. Furthermore, the argument for causality from dispersion relations in quantum field theory on both an experimental and theoretical level has been questioned by Bennett. ${ }^{4}$ He shows that dispersion relations for gammaaroton scattering are badly violated by experiment and that there are sound rasons why they should be. Furthermore, recent work by Thorne et al. ${ }^{3}$ at [a] Tech and Novikov et al. ${ }^{3}$ in Moscow show that Einstein's gravitational "Eld equations permit a new class of "traversable wormhole" (TW) solutions stinct from black hole and Einstein-Rosen bridge solutions. Provided that an exotic form of matter that permits superluminal energy flows can be fund, the TW's can be constructed and used for practical interstellar travel ${ }^{3}$ and for time travel to the past. A principle of "global self-consistency on closed timelike curves" is invoked to avoid the causal paradoxes that "change the past." Indeed, the position taken by Thome and Novikov et al. regarding csality paradoxes is very close to that taken independently in earlier drafts af this paper (e.g., 1.13 above).

Aharonov et al. ${ }^{11}$ have also published a gedanken experiment with quanIm retroactivity acting backwards in time. They also show how the superzesitions of weak forces can cause a strong force leading to a new kind of quantum amplifier. The general idea is that special superpositions of Juantum inputs can, though very rarely, produce an output far outside the inain of the inputs.

\subsection{Loops in Time}

The emerging new causality-violating paradigm can be glimpsed in King's er arks on "supercausal" loops in time. ${ }^{(7)}$ The above gedanken experiment is affered as a counterexample of claims that standard quantum mechanis does not allow decodable superluminal and retroactive messages using E-stein-Podolsky-Rosen spin-spin pair correlations. My claim is that the gen gedanken experiment gives causality-violating testable super-causal rechanisms that reproducibly generate "loops in time"2 of the third kind.

Loops in time of the first kind correspond to the use of traversable wormhries to move objects backwards in time on globally self-consistent closed z-relike world lines. Loops in time of the second kind use superluminal sals propagating energy on spacelike world lines. Loops in time of the -d kind use Einstein-Podolsky-Rosen-Bohm nonlocal quantum spin pair correlations to transmit decodable information backwards in time using only lightlike world lines for energy propagation. These loops of the third kind described in the gedanken experiment of this paper can be used for the local decoding in the past of useful messages transmitted from the future in a way that is free from time travel paradoxes. I must first define new types of signals that are not recognized by physics today.

The new notion of causality-violating traversable wornholes that can selfconsistently transport objects backwards in time (like the "flower from the future" in a story by Jorge Luis Borges called "The Flower of Coleridge," Other Inquisitions) would correspond to a loop in time of the first kind, since it takes place in the base space of the world geometry on closed timelike world lines.

If a man could pass through Paradise in a dream, and have a flower presented to him as a pledge that his soul had really been there, and if he found that flower in his hand when he awoke - Ay! - and what then? ... Wells' protagonist travels physically to the future. ... More incredible than a celestial flower or the flower of a dream is the flower of the future, the unlikely flower whose atoms now occupy other spaces and have not yet been assembled.

In contrast, the gedanken experiment in Sec. 1 would be the mechanism for the loop in time of the third kind described by another Borges story, "The Dream of Coleridge" (Other Inquisitions).

A thirteenth-century Mongolian emperor dreams a palace and then builds it according to his dream; an eighteenth century English poet (who could not have known that the structure was derived from a dream) dreams a poem abont the palace. In comparison with this symmetry, which operates on the souls of sleeping men and spans continents and centuries, the levitations, resurrections, and apparitions in the sacred books are not so extraordinary. ... Perhaps the series of dreams has no end, or perhaps the last one who dreams will have the key. ... Perhaps an archetype not yet revealed to men, an eternal object (to use Whitehead's term), is gradually entering the world; its first manifestation was the palace; its second was the poern. Whoever compared them would have seen that they were essentially the same.

In this paper I present highlights of new predictions in relativity. I will present a more detailed mathematical investigation of relativity without causality in a sequel to this paper. Relativity without causality is intended to be the new tangent group geometry for general relativity. My conjecture is that relativity without causality, which includes Hawking's "imaginary time,"12 is what is missing in the current attempts to make a coherent quantum gravity theory. I have adopted "the realistic philosophy of most working scientists"6 and have opted for the strategy of "dramatically revising our concept of space-time" in reconciling quantum nonlocality with relativity.

Causal signals of the first kind do not break the speed light barrier. However, causality-violating signals "of the second kind" do break the light barrier (see Fig. 6). Signals of the second kind transfer information by energymomentum flows that are somewhere, though not necessarily everywhere, spacelike outside of the local light cone.

Signals of the first and second kind have the common feature that they propagate energy and momentum along world lines of various types within space-time. There is still a third type of signal "of the third kind" using 


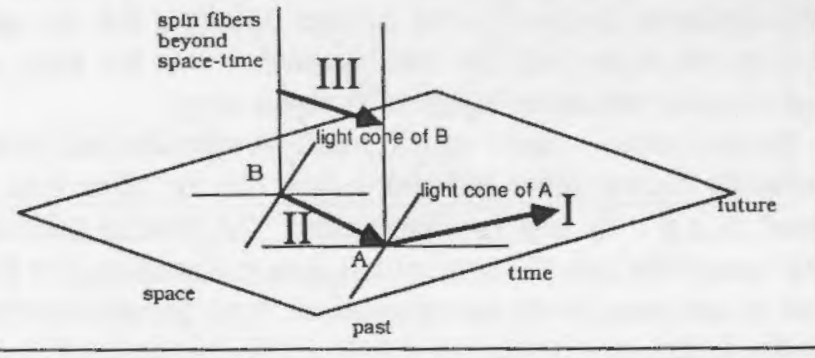

Figure 6. Fiber bundle picture of signals of first (I), second (II) and third (III) kind. I and II are modulated energy flows conveying messages. III is pure information flow conveying messages without corresponding energy flow.

controlled quantum nonlocality in fiber Hilbert space beyond space-time. This quantum signal does not propagate energy-momentum along world lines in space-time. Ne'eman ${ }^{5}$ has shown how nonlocal spin-spin correlations fit into the world fiber bundle picture. Space-time is the base space of the world fibered by Hilbert spaces of various kinds. In a quantum signal of the third kind local probabilities at space-time event A are controllably shifted from an arbitrarily separated space-time event $B$ because of the nonlocal fiber quantum connection between the detection processes at these two events.

The basic circuit for a precognitive quantum computer is the gedanken experiment described above. It, perhaps in a simplistic model of our own biocomputer, uses retroactive quantum signals of the third kind in accord with Hoyle's intuitions. ${ }^{2}$ These signals take the extradimensional route above and beyond space-time. These extra dimensions are part of the mathematics of fiber bundles used in the unified force theories. Thus signals of the third kind do propagate useful messages across space in the course of time, but they do so without propagating modulated energy-momentum as the cartier of that decodable information. The superluminal signaling gedanken experiment design given in this paper is a crucial objective test of these types of conjectures.

\subsection{New Causality-Violating Mass Shells?}

Relativistic quantum field theory describes real particles as "on the mass shell" and virtual particles as "off the mass shell." The mass shell corresponds to a pole in the complex energy plane in the integrand for the quantum field propagator. The boundary conditions correspond to the choice of a contour for the integral in the complex energy plane. The Feynman causal contour allows a Wick rotation of $90^{\circ}$ in the complex energy plane without crossing any obstructive singularities. This nice feature is lost in quantum gravity. The energy pole $E$ moves as the momentum $p$ changes. The basic forces (strong, electromagnetic, weak, gravity) are caused by virtual, not real, particles. Real photons are confined to the light cone, but spacelike virtual photons ase outside the light cone. Indeed, four-momentum conservation demands that the exchanged single virtual photon is outside the light cone. The commutator of the photon creation and destruction operators does not vanish outside the light cone. Since the photon has zero frame-invariant mass $m$, its Compton wavelength force range $\mathrm{h} / m c$ is infinite.

Real subluminal quantum particles on the mass shell are confined to timelike world lines inside the light cone. However, virtual subluminal particles off the mass shell are not confined to timelike world lines. They can be outside the light cone with exponentially damped probability of range equal to the Compton wavelength.

In contrast, real superluminal particles on a new branch of the mass shell are on spacelike world lines outside the light cone. Virtual superluminal particles can be inside the light cone also with exponentially damped probability. Thus both the subluminal and superluminal particles feel the Einstein barrier in real time. Subluminal particles obey causality, but superluminal particles do not.

Superluminal particles do not have a rest frame. They do have a transcendental frame in which their speed is infinite, their energy is zero, and their momentum is finite. They also have a special frame in which their speed is $\sqrt{2} c$ and their real gamma factor $\gamma=1 /\left[(v / c)^{2}-1\right]^{12}$ is 1 $(v / c>1)$. Gamma is greater than 1 for $c<v<\sqrt{2 c}$. Gamma is less than 1 for $v>\sqrt{ } 2 c$.

Superluminal particles lose energy as they accelerate. They time contract and length dilate into three-dimensional strings along their motion above $\sqrt{2} c$. Freely falling superluminal particles follow spacelike geodesics in pseudo-Riemannian curved space-time. Superluminal particles have quantum de Broglie waves that are shorter than their Compton wavelength. Their energy varies from zero to infinity.

We use the same special relativity formulas for superluminal particles as for subluminal particles. Only the gamma factor is different. The Lorentz transformations between observers in subluminal relative motion work for superluminal particles as well as subluminal particles and light. Although one can extend the group of Lorentz boosts in real time to include causalityviolating superluminal motions between observers, it will not be necessary to do so in this paper.

Signals of the second kind further subdivide into a short-wave superluminal tachyonic branch and a long-wave transluminal branch relative to the Compton wavelength $\mathrm{h} / m c$. I postulate that all elementary particles of frame-invariant mass $m$ have three possible modes of real existence characterized by three distinct mass shells for their relativistic quantum field propagators. The two new mass shells (i.e., energy poles of the propagator) are

$$
\begin{gathered}
E^{2}=p^{2} c^{2}-m^{2} c^{4}, \quad p>m c \text { (superluminal), } \\
E^{2}=m^{2} c^{4}-p^{2} c, \quad p<m c \text { (transluminal). }
\end{gathered}
$$

The superluminal short-wave branch represents signals of the second kind in Lorentzian space-time with spacelike flows of modulatable real energymomentum outside the local light cones.

\subsection{Transluminal Matter to Make Traversable Wormholes?}

The intelligent construction of a traversable wormhole requires a new phase of matter that violates causality in the form of "the weak energy condition." Just as the cosmic thermal microwaves are left over from the late stages of the big bang, this new causality-violating "transluminal phase" of dark matter may be a remnant of the "imaginary time" Riemannian quantum gravity era. Hawking puts the boundary condition that the universe "has no boundary" in this era of imaginary time. Visible matter, in a locally variably curved but globally spatially flat pseudo-Riemannian geometry, would only be $10 \%$ of the gravitating mass of the universe.

That is, the universe would have a dual-metric base space geometry on 
In of the topological, the projective and the affine connection levels of zmature of the geometry of the universe. The local tangent group would be -z than the traditional Lorentz group, since transluminal particles move n I izinary time rather than real time. Yet, translnminal particles must be $t^{2}=\mathrm{to}$ scatter off light, subluminal and superiuminal particles moving in el ane. This would imply a violation of local Lorentz invariance in the ane that local Lorentz invariance implied a violation of local Galilean ance in 1905.

-aginary time is just like real time and can be measured by our ordinary a minal clocks and radars. The distinction between real and imaginary the is topological as well as metrical. The signature of the transluminal meric is $(\mathrm{H}+\mathrm{H})$. Two ohservers in relative motion measuring the same rrsiuminal particle motion must use Euclidean rather than Lorentz frame - $=$ formations. The gamma factor for a transluminal frame transformation s

$$
\gamma=1 /\left[(v / c)^{2}+1\right]^{1 / 2}
$$

rostulate that a charged transluminal particle in the $(+1+)$ metric will - 1 real photon along a null geodesic in the dual Lorentzian (+H-) actric. The problem with traditional Lorentz frame invariance arises when z crosider a Feynman diagram in which transluminal world lines connect sit subluminal, luminal, and superluminal world lines. This does not zexent a retum to the Galilean subgroup of the Lorentz group, but to a sel higher group of the local tangent world geometry in which the Lorentz grap is a subgroup (see Sec. 2.4).

Femannian space-time world lines carry a local metric with positivefinite signature $(+\mathrm{H})$ in imaginary time that do not sense the Einstein rereic. These two metrics might be limiting cases of a still higher level metric on a hypercomplex manifold of the kind used in supersymmetry models. One metric has signature $+\mathrm{H}$, the other has signature $+\mathrm{H}$. I ther assume that null Lorentzian lightlike geodesics of signature + + rreat $+\mathrm{H}+$ signature transluminal world lines with $+\mathrm{H}$ - signature amelike and spacelike world lines. Einstein showed the independence of the -at of light, though not the frequency, on the relative speed between source ad absorber. This suggests that transluminal signals have modulatable real ry-momentum flows accessible to ordinary detectors whose world lines are restricted by the light cone barrier.

The Lorentz frame-dependent topological signature phase transition beween superluminal and transluminal particles occurs at zero energy, infinite peed, and finite Compton momentum $m c$. The Lorentz frames correspond z 1 subgroup of a higher curved base space (fiber bundle) local tangent pes symmetry group. That is, Lorentz invariance of the space-time interval s rolated locally in the presence of real transluminal matter. Lorentz invariance is already violated globally by general relativity. For example, there is a well-defined cosmological time since the big bang defined by the "Hubble 5 expansion of the universe and the condition that the cosmic photons shos an isotropic blackbody power spectrum with no redshifts and blueshifts. this newly predicted local violation of Lorentz symmetry is analogous to the action of Galilean invariance of absolute time by the higher symmetry of -rentz invariance. Note that the Lorentz invariant $d s^{2}$ is still frame invariat in the low-speed Galilean limit, but the Galilean invariant $d t^{2}$ is not me invariant in the Lorentzian regime of high speeds. There is a new higher symmetry frame invariant including both real and imaginary time.
This is not a descent to the lower symmetry group of Newtonian mechanics but, rather, an ascent to a higher symmetry group beyond the 1905 Einstein special relativity tangent geometry to the 1915 general relativity.

The transluminal long-wave branch occurs in what Hawking ${ }^{12}$ calls "imaginary time." Hawking pictures the preinflationary quantum gravity era as a compact four-dimensional metric space of signature $+1+$, which has a phase transition to a $+\mathrm{H}-$ signature metric space. I am suggesting that only about $10 \%$ of the +++ space becomes ++- . Thus $90 \%$ of the mass of the universe would still be in the primordial $+\mathrm{H}+$ transluminal phase and would account for the dark matter needed to make the universe spatially flat on the cosmological scale. Just as the three-degree cosmic microwaves are a fossil from the decoupling of matter from radiation in the later stages of the big bang, so the hypothetical transluminal dark matter would be a fossil from the earlier quantum gravity era.

Real transluminal particles move in imaginary time. Transluminal clocks time contract. Transluminal particles also stretch into strings along their direction of motion. They have a rest frame like subluminal particles and a transcendent frame like superluminal particles. Transluminal quantum de Broglie waves are longer than the Compton wavelength.

Freely falling transluminal particles follow (H+H) Riemannian geodesics. For example, suppose we have a Schwarzschild solution for the curved pseudo-Riemannian (+ +- ) space-time exterior to a spherically symmetric subluminal mass. The rule is to make a Wick rotation on the coordinate time from $d t$ to $i d t$ to compute the motion of the transluminal test particle; similarly for the motion of a subluminal or superluminal test particle in the positive-definite metric of a large transluminal source.

The cosmological equations should be modified to include a uniform distribution of transluminal particles at the critical density. This might account for the "great wall," the "great attractor," and for the mystery of galaxy formation and early quasars.

The matter-free unstable super-cooled vacuum used in the repulsive antigravity inflationary expansion of the early real-time $(\mathrm{HH})$ universe after it leaves the imaginary-time $(+H+)$ quantum gravity era is destroyed in a phase transition. The spin 0 pre-Higgs field goes superfluid developing a vacuum order parameter that destroys the effective cosmological constant driving the inflation. Free lepto-quarks and $\mathrm{X}$ particles are spat out in this phase transition to the GUT era. The quarks and colored gluons are confined later on when the electroweak force splits off from the strong force. The problem is that the pre-Higgs field must be tachyonic and bosonic in order to have the superfluid symmetry-breaking potential. If spin statistics is violated for tachyons, then the causality-violating pre-Higgs field must have spin $1 / 2$ rather than spin 0 in order to be bosonic. This can be considered as a test of my new theory. The subluminal causal bosonic spin 0 Higgs particles that give the $\mathrm{W}, \mathrm{Z}$ quarks and leptons their mass must be small vibrations in the spinor-bosonic pre-Higgs relativistic superfluid.

\subsection{Spin-Statistics Violation and Renormalization in Quantum Field Theory}

The two additional causality-violating mass shells are not recognized in today's relativistic quantum field theory. These new mass shells may make the current renormalization algorithm unnecessary. Fictitious particles with the wrong spin-statistics connection ${ }^{13}$ are used to regularize gauge theories. The spin-statistics connection is imposed by the requirement of causality and stable quantum vacua. Assuming the subluminal mass shell, Pauli ${ }^{14}$ showed that quantization of half-integer spinor fields with boson quantum statistics (i.e., commutators on creation and destruction operators) implies unstable 
quantum vacua (i.e., nonpositive-definite total field energy). The big bang and, possibly, quasars are examples of unstable quantum vacua. Quantization of integer spin fields with fermion statistics (anticommutators) implies superluminal energy propagation outside the light cone. Thus we must expect that the new phases of matter will have the wrong spin-statistics connection. That is, causality-violating particles will be scalar fermions, spinor bosons, vector fermions, etc. Pauli's 1941 proof of the connection between spin and statistics rested on the assumption of causality (i.e., denial of superluminality) and stability of the quantum vacuum. Neither is really true. I have already mentioned the work of Bennett on the breakdown of dispersion relations. As another example, the vacuum of the early universe is not stable. Thus spontaneous symmetry-breaking is a vacuum phase transition triggered by an instability in which a fluctuation is chaotically amplified to macroscopic dimensions.

Subluminal particles obey the spin-statistics connection, that is, spin 0 (scalar), 1 (vector), and 2 (tensor) are bosons that can occupy the same quantum state. Spin 1/2 (spinor) and 3/2 (spinor-vector) are fermions that obey the Pauli exclusion principle of no more than one fermion per quantum state.

I postulate that superluminal particles violate the spin-statistics connection. That is, scalar spin 0 and vector spin 1 superluminal particles (tachyons) are fermions. They are needed to remove the infinities from the subluminal sector of quantum field theory which is incomplete without them (e.g., Pauli-Villars regularization in QED and Faddeev-Popov "ghosts" in non-Abelian gauge theory). Quantum corrections come from Feynman diagrams with loops of virtual particles that cause ultraviolet infinities in causal quantum field theory. Including loops of virtual superluminal shadow particles with the opposite spin statistics might make the quantum corrections finite without going to extra dimensions. Indeed, in the path integral formulation it is necessary to go to the Euclidean signature ( $+1+$ ) for a proper definition of a convergent path integral. Perhaps, in that case, one should use only transluminal particles so that there is a finite ultraviolet cutoff at $m c^{2}$ ? In my view, superluminal and transluminal particles are not fictitious, and they have the same frame-invariant mass as their sublumiual partners. Indeed, a uniform cosmological distribution of very cold, slowly moving neutral real transluminal particles left over from the quantum gravity era before the first $10^{-43} \mathrm{~s}$ may well account for $90 \%$ of the matter in the universe. An alternative, wild idea is that the missing mass is not in the form of real particles at all, but corresponds to a transluminal cosmological constant giving a finite energy to an exactly spatially flat vacuum as the length scale approaches the Hubble radius. Implicit in these considerations is another wild idea that the world quantum geometry has a nondifferentiable fractal structure that has self-similar scale invariant effects all the way up from below the ultramicro Planck scale to the cosmological Hubble scale. Penrose ${ }^{1}$ has pointed out that the transition from the quantum to the classical limit has some surprises. An extension of classical general relativity to the dual metric is required to properly formulate these speculations.

Another reason why causality-violating real particles are a good candidate for at least some of the missing mass of the universe is that the Pauli exclusion principle does not operate for superluminal and transluminal electrons, protons, and neutrons. Therefore, if there are high-density concentrations of transluminal stuff, there would be no diverse and complex many-particle nuclear, atomic, and molecular shell structures to support most of the normally observed electromagnetic quantum jumps. We would still expect to see hydrogen spectra, but they would be superluminally and transluminally
Doppler shifted. Astronomers would misinterpret what they were seeing for lack of the proper theory.

\subsection{Quantum Time Operator Requires Causality Violation}

Up until now, because of the energy gap $\left(-m c^{2}\right.$ to $\left.+m c^{2}\right)$, it has been impossible to define a relativistic quantum time operator canonically conjugate to the energy operator as normally required by the uncertainty principle in commutator form. Adjunction of the new causality-violating mass shells fills this energy gap, making it possible to consistently define a quantum time operator. Therefore, superluminal violation of causality is necessary for the proper definition of time at the quantum level.

For example, consider the momentum-position uncertainty relation which follows from the fact that the commutator of the two canonically conjugate Hermitian operator observables (in boldface below) is $i$ h and the orthocompleteness of the eigenstates for each. That is,

$$
\begin{gathered}
{[\mathbf{q}, \mathbf{p}]=i \mathrm{~h},} \\
\mathbf{p}|p\rangle=p|p\rangle, \\
1=\Sigma_{p}|p\rangle\langle p|, \\
\mathbf{p}=\Sigma_{p} p|p\rangle\langle p|, \\
\mathbf{q}|q\rangle=q|q\rangle, \\
1=\Sigma_{q}|q\rangle\langle q|, \\
\mathbf{q}=\Sigma_{q} q|q\rangle\langle q|, \\
(\Delta q)^{2}(\Delta p)^{2} \geq(1 / 4)\langle-i[\mathbf{q}, \mathbf{p}]\rangle^{2}=h^{2} / 4
\end{gathered}
$$

Orthocompleteness requires

$$
\begin{gathered}
\left\langle p^{\prime} \mid p\right\rangle=\delta\left(p^{\prime}-p\right), \\
\left\langle q^{\prime} \mid q\right\rangle=\delta\left(q^{\prime}-q\right), \\
\langle q \mid p\rangle=\exp (i 2 \pi p q / \mathrm{h}), \\
|p\rangle=\Sigma_{q}|q\rangle\langle q \mid p\rangle, \\
|q\rangle=\Sigma_{p}|p\rangle\langle p \mid q\rangle .
\end{gathered}
$$

The essential point is that in order to obey these equations, the eigenvalue spectrum of the Hermitian operators for a free particle with infinite space boundary conditions must be continuous from $-\infty$ to $+\infty$ with no gaps. Finite space standing-wave boundary conditions give a discrete momentum eigenvalue spectrum. For the case of the $z$ component of orbital angular momentum the conjugate azimuthal angle obeys periodic boundary conditions and an uncertainty relation is still possible because $L$ can go negative. When the subluminal particle is not free, there is also a discrete energy eigenvalue spectrum of bound states which is negative when the rest mass is subtracted out. For the relativistic subluminal free particle, we have negative energies, 
It there is a rest mass gap from $-m c^{2}$ to $+m c^{2}$. This means that we carnot define orthocomplete eigenstates of the relativistic frame-dependent une operator $\mathbf{t}$ that is canonically conjugate to the energy operator $\mathbf{E}$. That is. cannot write

$$
\begin{gathered}
\mathbf{E}|E\rangle=E|E\rangle, \\
1=\Sigma_{E}|E\rangle\langle E|, \\
\mathbf{E}=\Sigma_{E} E|p\rangle\langle p|, \\
\mathbf{t}|t\rangle=t|t\rangle, \\
1=\Sigma_{t}|t\rangle\langle t|, \\
\mathbf{t}=\Sigma_{t} t|t\rangle\langle t|, \\
\langle\Delta)^{2}(\Delta E)^{2} \geq(1 / 4)\langle-i[\mathbf{t}, \mathbf{E}]\rangle^{2}=h^{2} / 4, \\
\left\langle E^{\prime} \mid E\right\rangle=\delta\left(E^{\prime}-E\right), \\
\left\langle t^{\prime} \mid t\right\rangle=\delta\left(t^{\prime}-t\right), \\
\langle t \mid E\rangle=\exp (i 2 \pi E t / h), \\
|E\rangle=\Sigma_{t}|t\rangle\langle t \mid E\rangle, \\
|t\rangle=\Sigma_{E}|E\rangle\langle E \mid t\rangle .
\end{gathered}
$$

Note that the Fourier integral representation of the Dirac delta function in tme requires that the domain of the integration energy variable $E$ have no saps in it. This is the essential reason why a relativistic time operator $\mathbf{t}$ cannot be defined, and why the true nature of the energy-time uncertainty relation ${ }^{(10)}$ has remained controversial to this day,

$$
\delta\left(t^{\prime}-t\right)=\int_{-\infty}^{+\infty} \exp (i 2 \pi E t / \mathrm{h}) d E .
$$

The simple qualitative point I wish to raise here is that including the superluminal mass shell widens the space of energy eigenfunctions and fills the subluminal mass gap. In this sense a proper definition of time as an observable property of massive particles in relativistic quantum mechanics seems to demand causality violation. (A time operator for the massless photon can apparently be defined without causality violation.)

\subsection{Contra Dispersion Relations}

Dispersion relations connect the real and imaginary parts of the scattering amplitude as a function of energy. They follow from the Cauchy integral theorem and the Titchmarsh theorem. It is the Titchmarsh theorem that is thought to be the link with causality in the form that the scattered wave cannot be emitted before the incident wave reaches the scatterer. Closer analysis reveals causality to be only a sufficient condition and not a necessary one to apply the Titchmarsh theorem. One can still get the dispersion relations even if the scattered wave is precognitively emitted before the arrival of the incident wave. Physics graduate students are taught that dispersion relations in particle scattering are a theoretical consequence of causality and that experiments confirm them. Neither is true.

Bennett ${ }^{4}$ has shown that dispersion relations are violated by experiment and that they are not an adequate proof for causality. Bennett also shows that the original Wheeler-Feynman action-at-a-distance electrodynamics is finite at the quantum level and has retroactive quantum vacuum fluctuations with future causes.

The physics of massless light is not changed by my new theory. Only the physics of massive particles is changed. It does suggest, however, that the agreement between experiment and theory for the dispersion relations in collisions involving massive particles is, at best, only approximate. Dispersion relations should be violated when Feynman "ghost" diagrams, now properly reinterpreted as the coupling of subluminal particles with both superluminal and transluminal particles, can no longer be neglected.

The interpretation of Kramers-Kronig relations for light as evidence for causality is logically erroneous. That is, the Kramers-Kronig relations are consistent with aduanced potentials operating retroactively with a finite range $\tau_{0}$ from the future to the present. This can be seen from a reexamination of the standard argument for causality, for example, by Pais. (11)

'Thus, suppose a 'cause' $C$ at time $t-\tau$ contributes to an 'effect' $E$ at time $t$, and that $C$ and $E$, are linearly related:

$$
E(t)=\int_{-\infty}^{\infty} F(\tau) C(t-\tau) d \tau
$$

Causality, ' $E$ cannot precede $C$ ', is expressed by

$$
F(t)=0 \quad t<0 .
$$

A general mathematical theorem says that this condition is equivalent to the following two statements: the Fourier component $G(\omega)$ defined by

$$
G(\omega)=\int_{-\infty}^{\infty} F(t) e^{i \omega t} d t
$$

can be continued analytically to complex values of $\omega$ with $\operatorname{Im} \omega>0$, and has no singularities in this region; and $G(\omega)$ satisfies

$$
\operatorname{Re} G(\omega)=\frac{1}{\pi} P \int_{-\infty}^{\infty} \frac{\operatorname{Im} G\left(\omega^{\prime}\right)}{\omega-\omega^{\prime}} d \omega^{\prime} . "
$$

However, look at (72); since the integral in (73) is over an infinite amount of time, it makes no difference mathematically to the Kramers-Kronig protodispersion relation $(74)$ if we replace " $\tau<0$ " in (74) by " $\tau<-\tau_{0}$," i.e.,

$$
F(\tau)=0, \quad \tau<-\tau_{0},
$$

where $\tau_{0}$ is the range of the "foreknowledge." Let us not forget that Dirac needs foreknowledge to eliminate the runaway solutions in a consistent classical theory of radiation reaction. Thus it is clear that the validity of Kramers-Kronig relations for light does not logically demand cansality. That is, causality is a sufficient condition for dispersion relations, but it is not a necessary condition. I quote, however, an objection to this argument raised by the referee: "Choosing a specific $\tau_{0}$ appears to imply a fixed retrospective 
time factor throughout the universe, but tachyons imply arbitrarily large reversals. This would appear to require moving $\tau_{0}$ in the limit to $-\infty$ causing some problems." However, the work of Bennett ${ }^{4}$ is germane to this point.

\subsection{Boundary Conditions for Tachyonic Propagator}

Energy $E$ and momentum $p$ are mathematically real in terms of the motion of particles and waves (accessible to our detectors) for both the short-wave superluminal and the long-wave transluminal mass shells of the causality-violating phases of matter. The boundary conditions on the superluminal propagator, for example, are the opposite of Feynman's causal boundary conditions for real subluminal particles on the mass shell. Virtual particles are off the mass shell and correspond to the incoherent noise in the coherent signal which is the real particle pole of the propagator.

Thus, according to Keynman, subluminal particles propagate positive energy forwards in time and negative energy backwards in time. Antiparticles moving forward in time with positive energy are then equivalent to particles moving backwards in time with negative energies and opposite intemal quantum numbers.

In contrast, short-wave superluminal particles, that is, "tachyons," (12) propagate positive (negative) real energy backwards (forwards) in time. One can then define antitachyons in Feynman's sense. The violation of Feynman's boundary condition is proved by starting with a tachyon in the transcendent frame $\mathrm{K}$ of infinite velocity, say in the $+z$ direction where $E=0$ and $p_{z}=m c$. Then make an ordinary Einstein subluminal boost of $v<c$ in the $+z$ direction to the frame $\mathrm{K}^{\prime}$. The velocity addition law tells us that the tachyon is moving in the $-z^{\prime}$ direction with superluminal speed $u^{\prime}=c^{2} / v$. From this it follows that the energy $E^{\prime}$ of the tachyon in the $\mathrm{K}^{\prime}$ frame is positive:

$$
E^{\prime}=+m c^{2} /\left[u^{2} / c^{2}-1\right]^{1 / 2} .
$$

However, the sub-boost in space-time shows that the tachyon of positive energy must move backwards in time in the $\mathbf{K}^{\prime}$ frame. $\mathrm{K}^{\prime}$ was sub-boosted parallel to the direction of the tachyon's motion in the transcendent $\mathrm{K}$ frame (where all points on the tachyon world line are simultaneous, i.e., $d t=0$ ). Similarly, if the sub-boost to $\mathbf{K}^{\prime}$ is antiparallel to the tachyon's motion in the transcendent frame $\mathrm{K}$, we get a negative energy tachyon moving forwards in time. Indeed, this will destabilize the vacuum, but we may have evidence from violent astrophysical phenomena that the vacuum is not stable. There must be some sort of nonlinear saturation damping out the emission of real tachyons of negative energy propagating forwards in time.

The short-wave superluminal free particle eigensolutions do not form a complete set because of the momentum gap. However, when the longwave transluminal eigensolutions are added to them, we do get a complete set. Therefore, conservation of probability in causality-violating relativistic quantum field theory (yet to be formulated in detail) is assured.

\subsection{Quantum Spin Thermodynamics Mind-Matter Model}

Suppose that the physical substrate of mind is an intelligent quantum spin switching network making a non-Boolean logic quantum computer. The spins could be that of the weakly bonding lone protons (e.g., electropositive hydrogens in water) and perhaps unshared electron pairs and mobile $\pi$ electrons in complex biomolecules. The second law of thermodynamics implies that when a "hot" negative quantum spin temperature

$$
T_{\text {spin }}<0
$$

is coupled to a "cold" positive lattice temperature $T_{\text {lattice }}>0$ in a Carnot heat engine, heat $Q$ flows from both spin and lattice reservoirs and is entirely converted to mechanical work $W$. That is, the efficiency $W / Q_{\text {spin }}>1$. There is no waste heat. Furthermore, a tiny heat flow $Q_{\text {spin }}>0$ from the negative spin temperature network can trigger a much larger conversion of heat $Q_{\text {lattice }}<0$ to work $W$ from the positive temperature lattice. Thus

$$
\begin{aligned}
W / Q_{\text {spin }} & =\left[Q_{\text {spin }}-Q_{\text {latrice }}\right] / Q_{\text {spin }}=1+\left|Q_{\text {latrice }} / Q_{\text {spin }}\right| \\
& =1+T_{\text {lattice }} /\left|T_{\text {spin }}\right|>1 .
\end{aligned}
$$

For example, let $T_{\text {spin }}=-0.1 \mathrm{~K}$ and let $T_{\text {latice }}=300 \mathrm{~K}$. The quantum heat engine efficiency is then $3000 \%$. This is not a violation but a quantum "loophole"15 in the second law of thermodynamics - a counterintuitive consequence of it when combined with the quantum principle. Is this how the mind moves matter, the mind being the spin system and the lattice being the nerve cell system in which $W$ triggers a nerve pulse for a relatively tiny quantum spin energy flow?

The above considerations are compatible with the view of Sperry ${ }^{16}$ : "the conscious phenomena of subjective experience do interact on the brain process exerting an active causal influence ... the contents of subjective mental experience are recognized as important aspects of reality in their own right, not to be identified with neural events as these bave bave beretofore been conceived, nor reducible to neural events. [emphasis added] ... "the subjective mental properties and phenomena are posited to have top-level control as causal determinants. On these terms mind moves matter."

\subsection{The Relativistic Rocket Problem and Practical Interstellar Travel}

Thome et al. have shown that practical interstellar travel would be possible if traversable wormholes could be found or manufactured with matter (possibly transluminal) violating the "weak energy condition." Even without traversable wormholes, however, there is another possibility for practical interstellar travel. Let us consider the textbook "relativistic rocket" problem in the context of this theory. Imagine propelling the relativistic rocket along a subluminal timelike $1 g$ hyperbolic world line. Use a "fuel" capable of superluminal local exhaust speed $u$ along spacelike or Riemannian world lines for tachyonic or transluminal fuel particles, respectively. The energy and momentum conservation laws imply that the ratio of final to initial mass $M(\tau) / M(0)$ required for such a trip of proper time $\tau$ along the timelike hyperbolic world line of the rocket has an exponential dependence on the negative reciprocal of the propellent superluminal exhaust speed $u$. That is,

$$
M(\tau) / M(0)=\exp (-g \tau / u) .
$$

The limit of $M(\tau) / M(0)$ as $u \rightarrow \infty$ is 1 . Therefore, the energy efficiency is only limited by how fast the propellent can be ejected beyond the light barrier. These considerations coupled with those of Thome et al. on traversable wormholes should profoundly modify the SETI (Search for Extra-Terrestrial Intelligence) program.

\section{Acknowledgment}

I am primarily indebted to my old Cornell professor, Philip Morrison, for an indelible grand vision of physics that bridged C.P. Snow's "Two Cultures" in the 1950s. I have also been stimulated by direct contact with the late 
Richard Feynman (1963 and 1969), David Bohm (1971), and Abdus Salam (1973). Of course, I am greatly indebted to Professor Henry Stapp (Lawrence Berkeley Lab), Professor Peter Bussey (University of Glasgow), Professor Oreste Piccioni (UCSD), and Dr. Nick Herbert. I would also like to acknowledge important technical contributions by Creon Levit (NASA Ames) who insisted that it should be possible to make a superluminal signaling device in a simpler way than a double-slit arrangement by using a simple interferometer. A double-slit arrangement involves a more complicated spread of phases. The interferometer has basically a sharp phase with some noise. Should experiments confirm my prediction, then Herbert, Stapp, Bussey, Piccioni, and Levit deserve some of the credit, although none of the blame should it fail. Professor Waldyr Rodrigues of Instituto Matematica, UNICAMP (Brazil), showed me some unpublished work by Andrei Sakharov on transluminal particles in the big bang in 1985 when I was a visiting professor. I would like to thank Dr. David Sarfatti for editing help, Jagdish Mann and Csaba Szabo (Lt. Col. USAR Special Forces), and the U.S. Navy (PACE) for financial support. I also thank Stephen Schwartz and A. Lawrence Chickering of the Institute for Contemporary Studies for their support. Finally, I thank Kim Burrafato for bringing the Optics Guide to my attention at a significant moment.

\section{APPENDIX: EFFECT OF TRANSMITTER INTERFEROMETER REFLECTION PHASE SHIFTS ON THE RECEIVER SIGNAL}

Let us consider the effect of variable reflection phase shifts at the mirrors of the transmitter interferometer on the local decoding of the superluminal retroactive quantum spin-spin signal at the distant receiver. A reflection phase shift of $\delta=180^{\circ}$ rather than $\delta=90^{\circ}$ [Sec. 1.7, Fig. 3, Eqs. (23) to (30)], appears to make Stapp's second objection obviously irrelevant. First, I will justify the use of the $90^{\circ}$ phase shift for total internal reflection. Second, I will consider the effect of the $180^{\circ}$ phase shift for external reflection, which considerably simplifies matters and provides a strikingly clear counterexample to Stapp's arguments against faster-than-light signaling in standard quantum mechanics.

"External reflection is defined as reflection at an interface where the incident beam originates in the material of lower refractive index. ... Intemal reflection refers to the opposite case."(13a) "During extemal reflection, the light waves undergo a $180^{\circ}$ phase shift. No phase shift occurs for internal reflection (except in the case of total intemal reflection)."(13b)

External reflection away from normal incidence is generally inefficient (e.g., about $4 \%$ reflectance at normal out to $20^{\circ}$ incidence angle $\xi$ in air to polished optical glass with glass index of 1.52 for polarizations both parallel and perpendicular to plane of incidence). In contrast, internal reflection becomes total (i.e., 100\% reflectance) for both polarizations above a critical angle of $41^{\circ} 12^{\prime}$ when the ratio of indices is again $n=1.52$. For example, the incident beam originates in the glass for total reflection at the air/glass boundary.

What is the reflection phase shift $\delta$ in the region of total internal reflection? The boundary conditions to Maxwell's electromagnetic field equations between two dielectrics give the Fresnel equations [e.g., Eqs. (11-51) to (11-54), Ref. 14, p. 199]:

$$
\left(\sin ^{2} \xi-n^{-2}\right)^{1 / 2} / \cos \xi=\tan (\delta / 2)
$$

if the electric vector is perpendicular to the plane of incidence. If the electric vector is parallel to the plane of incidence, then

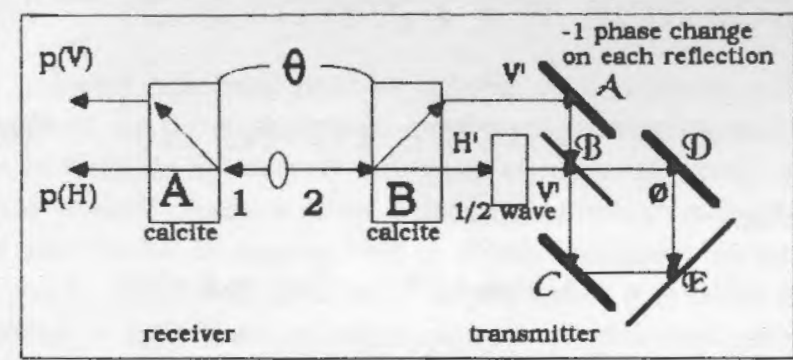

Figure 7. The gedanken experiment. FTL signal $=\sin (2 \theta)[1-\cos \phi]$ which survives even if $\langle\cos \phi\rangle=0$.

$$
\left(\sin ^{2} \xi-n^{-2}\right)^{1 / 2} / n^{-2} \cos \xi=\tan \left(\delta^{\prime} / 2\right) .
$$

Figure 3 and the discussion of Sec. 1.7 assumed that $\delta=90^{\circ}$. This requirement complicates the design of the interferometer, because the light beams must move through a dielectric medium whose index of refraction is larger than that of the mirrors. This is not a difficulty of principle. Thus the phase shift of $i$ used in Eqs. (23) to (30) in Sec. 1.7 corresponds to, for example,

$$
\begin{gathered}
\left(\sin ^{2} \xi-n^{-2}\right) / \cos ^{2} \xi=1, \\
\sin ^{2} \xi=\left[1+n^{-2}\right] / 2,
\end{gathered}
$$

for polarization perpendicular to the plane of incidence. Similarly,

$$
\begin{gathered}
\left(\sin ^{2} \xi-n^{-2}\right)^{1 / 2} / n^{-2} \cos \xi=1, \\
\sin ^{2} \xi=2 /\left(n^{2}+1\right), \quad n^{2}>1,
\end{gathered}
$$

for polarization parallel to the plane of incidence.

Thus it is possible to achieve a reflection phase shift of $i$ if we assume, as I have done, that the quantum probability amplitude phase shifts like the classical electric vector does. the motivation for using $i$ was high reflection field intensity efficiency. However, if we relax that requirement, supposing an intense laser pumped pair source, we can use external reflection in which $\delta=180^{\circ}$ and still get a good signal-to-noise ratio at the receiver (Fig. 7). This design choice also makes the transmitter interferometer much easier to build. Equations (23) to (30) now become

$$
\begin{gathered}
\mathrm{I}=\left\langle 1,2 \mid V_{1}, V_{2}^{\prime}, \mathcal{A}, \mathcal{B}, \mathcal{D}, \mathcal{E}\right\rangle=-(1 / 2) \cos \theta e^{i \phi(2)}, \\
\mathrm{II}=\left\langle 1,2 \mid V_{1}, H_{2}^{\prime}, \mathcal{B}, \mathcal{D}, \mathcal{E}\right\rangle=-(1 / 2) \sin \theta e^{i \phi(2)}, \\
\mathrm{III}=\left\langle 1,2 \mid V_{1}, V_{2}^{\prime}, \mathcal{A}, \mathcal{B}, \mathcal{C}, \mathcal{E}\right\rangle=+(1 / 2) \cos \theta, \\
\mathrm{IV}=\left\langle 1,2 \mid V_{1}, H_{2}^{\prime}, \mathcal{B}, \mathcal{C}, \mathcal{E}\right\rangle=+(1 / 2) \sin \theta, \\
\mathrm{V}=\left\langle 1,2 \mid H_{1}, V_{2}^{\prime}, \mathcal{A}, \mathcal{B}, \mathcal{D}, \mathcal{E}\right\rangle=+(1 / 2) \sin \theta e^{i \phi(2)}, \\
\mathrm{II}=\left\langle 1,2 \mid H_{1}, H_{2}^{\prime}, \mathcal{B}, \mathcal{D}, \mathcal{E}\right\rangle=-(1 / 2) \cos \theta e^{i \phi(2)},
\end{gathered}
$$




$$
\begin{gathered}
\mathrm{VII}=\left\langle 1,2 \mid H_{1}, V_{2}^{\prime}, \mathcal{A}, \mathcal{B}, \mathcal{C}, \mathcal{E}\right\rangle=-(1 / 2) \sin \theta \\
\mathrm{VIII}=\left\langle 1,2 \mid H_{1}, H_{2}^{\prime}, \mathcal{B}, \mathcal{C}, \mathcal{E}\right\rangle=+(1 / 2) \cos \theta
\end{gathered}
$$

Therefore,

$$
\begin{aligned}
\mathrm{I}+\mathrm{II}+\mathrm{III}+\mathrm{IV}= & -(1 / 2) \cos \theta e^{i \phi(2)}-(1 / 2) \sin \theta e^{i \phi(2)} \\
& +(1 / 2) \cos \theta+(1 / 2) \sin \theta \\
=+(1 / 2)\{\cos \theta+\sin \theta\}\left[1-e^{i \phi(2)}\right], & \\
\mathrm{V}+\mathrm{VI}+\mathrm{VII}+\mathrm{VIII}= & +(1 / 2) \sin \theta e^{i \phi(2)}-(1 / 2) \cos \theta e^{i \phi(2)} \\
& -(1 / 2) \sin \theta+(1 / 2) \cos \theta \\
= & \left.+(1 / 2)\{\cos \theta-\sin \theta\}\left[1-e^{i \phi(2)}\right], \quad \text { A } 55\right) \\
|\mathrm{I}+\mathrm{II}+\mathrm{III}+\mathrm{IV}|^{2}= & (1 / 4)\{1+2 \cos \theta \sin \theta\}\left|1-e^{i \phi(2)}\right|^{2} \\
= & (1 / 4)\{1+\sin 2 \theta\}\left|1-e^{i \phi(2)}\right|^{2} \\
= & (1 / 2)\{1+\sin 2 \theta\}(1-\cos \phi) .
\end{aligned}
$$

Similarly,

$$
\begin{aligned}
& |\mathrm{V}+\mathrm{VI}+\mathrm{VII}+\mathrm{VIII}|^{2}=(1 / 4)\{1-2 \cos \theta \sin \theta\}\left|1-e^{i \phi(2)}\right|^{2} \\
& =(1 / 4)\{1-\sin 2 \theta\}\left|1-e^{i \phi(2)}\right|^{2} \\
& =(1 / 2)\{1-\sin 2 \theta\}(1-\cos \phi) \text {. }
\end{aligned}
$$

I claim that Eqs. $\left(31^{\prime}\right)$ and $\left(32^{\prime}\right)$ make Stapp's second argument obviously false. The essence of Stapp's second argument (e.g., Sec. 1.9) is that we must sum over all $\phi$ to compute the local receiver photon detection probabilities. For example, Stapp writes:

The failure of the locality property in ... quantum theory ... does not contradict Einstein's principle that no signal travels faster than light. For by a signal is meant a controllable transfer of information - a message. Within the structure of these formalisms no such con- trolled faster-than-light information transfer is possible. This follows immediately from the fact that whereas within the quantum formalism (or the classical formalism) the probability of a specified result in one region, subject to the condition of a specified result in the other region, depends in general on the latter specification, and hence on the experimental setting in the other region, nevertbeless a summation over all passible results of the experiment in the other region with proper weights gives a result that is independent of the choice of the experimental setting in that region. This entails that there is no predictable dependence of the observations in one region upon the choice of setting in the other. ${ }^{(15)}$

However, the above model is a counterexample to Stapp's claim, because even if we suppose that the sum of $\cos \phi$ "with proper weights" $\rho(\phi)$ in the transmitter region is done, and further suppose that my idea of the coberent subensemble of Secs. 1.9 and 1.10 is incorrect,

$$
\langle\cos \phi\rangle=\int \cos \phi \rho(\phi) d \phi=0 ;
$$

nevertheless, there is still a "predictable dependence of the observations" in the receiver region "upon the choice of setting" $\boldsymbol{\theta}$ in the "other" transmitter region using only standard quantum mechanics. Indeed, the superluminal (and retroactive in delayed choice mode) signal $S$ has the Josephson tunneling currentlike form

$$
S=p(V, \theta)-p(H, \theta)=\sin 2 \theta,
$$

in which the misalignment $\theta$ between the calcites across an arbitrary four-dimensional space-time separation between the two detections of the photons in the same individual pair is analogous to the phase difference of the superconducting order parameter across a tiny nomal barrier.

I am assuming that the quantum wave function is complete in describing individual quantum systems and is not merely a description of ensembles. The fact that we can now observe individual trapped ions and that they conform to quantum predictions in their photon interactions seems to falsify the strictly ensemble interpretation of the wave function.

Received 12 February 1990. 


\begin{abstract}
Résumé
On propose un gedanken experiment pour un appareil de signalization supraluminale (FTL) utilisant des paires photoniques correlées en polarization emises en séquence. Seules les règles de Feynman de mécanique quantique standard sont utilisées. La métbode nouvelle consiste à laisser le photon transmetteur intéragir avec soi même. Il est alors passible de transmettre et de décoder localement des signaux supraluminaux par le deplacement, contrôlable, des probabilités quantiques de polarization du photon à travers une separation arbitraire d'espace-temps entre les détections des deux photons de la même paire. Le signal supraluminal est encodé avec le message en tournant d'un angle $\theta$ un détecteur interférométrique de transmission en calcite par rapport à un recepteur fixe en calcite, ou, en alternative, en changeant la phase moyenne $\phi_{0}$ de l'interféromètre. Par conséquant, le message est décodé en mesurant le degré changeant de polarisation linéaire de la lumière du récepteur. Le principe de causalité, que les effets se passent toujours après les causes en tout référentiel, sera pris en défaut si cette expérience se passe comme prévu. Les objections des paradoxes causaux et les demonstrations interdisant les signaux supralumineux sont commentés aussi. La deuxième partie de cet article entame l'étude de l'effet de la violation de la causalité sur le reste de la physique. Par exemple, eventuelles violations de la connection spin statistique et l'invariance locale de Lorentz de la matière noire, un opérateur quantique relativiste, un nouvel aperçu des infinis dans la théorie quantique de champs, trous de vers traversables comme machines du temps, et thermodynamiques quantigue du spin dans l'intéraction pensée-matière sont parmi les sujets traités.
\end{abstract}

Pindinotes

2. Penrose, The Emperor's New Mind (Oxford, 1989). "From the first if the above experiments, we seem to deduce that conscious action takes smething like a second to a second and a half before it can be effected, wile according to the second experiment, consciousness of an external eent does not seem to occur until a half second after that event has aken place. Imagine what happens when one responds to some unanscipated external occurrence. Suppose that the response is something then requires a moment's conscious contemplation. It would appear, on the basis of Libet's findings, that half a second must elapse before consoiousness is called into play; and then, as Kornhuber's data seem to inply, well over a second is needed before one's 'willed' response can ulie effect. The whole process from sensory input to motor output, would sem to require something like two seconds! The apparent implication of these two experiments taken together is that consciousness cannot even be called into play at all in response to an external event, if that response is to take place within a couple of seconds or so! ... Can we take these experiments at face value? If so we appear to be driven to the conclusion that we act entirely as 'automatons' when we carry out any action that sould take less than a second or two in which to modify a response [p. 442] ... Perhaps consciousness is, after all, merely a spectator who experiences nothing but an 'action replay' of the whole drama. Similarly, on the face of it, there would, on the basis of the above findings, be no ime for consciousness to be playing any role at all when, for example, one plays a shot at tennis - and certainly not at ping-pong! ... But that consciousness should be playing no role at all in the decision as to what shot should be played at the time is something that I find a little hard bo credit ... Such comments would be even more pertinent in relation to ordinary conversation. Again, although one might partly anticipate what the other would be saying, there must often be something unexpected in the other's remarks, or conversation would be entirely unnecessary! It certainly does not take as much as a couple of seconds to respond to someone in the normal way of conversation! ... I should like to make an alarming suggestion ... I suggest that we may actually be going badly wrong when we apply the usual physical rules for time when we consider consciousness! There is, indeed, something very odd about the way that time actually enters into our conscious perceptions in any case, and I think that it is possible that a very different conception may be required when we try to place conscious perceptions into a conventionally time-ordered framework. Consciousness is, after all, the one phenomenon that we know of, according to which time needs to 'flow' at all! [p. 443] ... My guess is that ... the time of our perception does not 'really' flow in quite the linear forward-moving way that we perceive it to flow ... [p. 444] ... Suppose that there is even something vaguely teleological about the effects of consciousness, so that a future impression might affect a past action. Surely this would lead us into a contradiction, like the paradoxical implications of faster-than-light signalling that we considered - and justly ruled out [cf. p. 212] ...?" [p. 445]

If one is ready to violate causality, as I am, but Penrose is not, then his line of inquiry can be extended beyond where he is ready to go. It suggests, in the light of Hoyle's remarks (Endnote 2) that the assumption of morally responsible "free will" demands teleological quantum retroactivity in ordinary consciousness; otherwise, the measured time delay between our motor actions and our awareness of them would doom us to the status of mere automatons helplessly observing what we 
do after we have done it. On the other hand, Penrose is not yet ready to embrace causality violation completely. He prefers a direct contact of consciousness with "Platonic reality" which vaguely evades the real issue, in my opinion. On the strong anthropic principle (see Endnote 2) and a literal interpretation of Wheeler's "self-excited participatory universe," Penrose, not liking controllably retroactive future causes very much, remarks on p. 295: "It is our own presence which, on this view, conjures our past into existence. The circularity and paradox involved in this picture has an appeal for some, but for myself I find it distinctly worrisome - and, indeed, barely credible." Penrose's view here is understandable, since violation of causality would invalidate many of the Hawking-Penrose classical black hole singularity theorems. However, I do agree with Penrose's remark about the many-worlds interpretation of quantum mechanics on p. 296: "I do not see why a conscious being need be aware of only 'one' of the alternatives in a linear superposition."

Penrose points out a peculiar feature of the classical limit of quantum mechanical computers. It should be borne in mind that the information strings in a quantum spin computer are quantum states with large spin. Even if the quantum computer is made of a large number of other kinds of nonlocally correlated two-state systems, the mathematical structure of the strings will be isomorphic to the model Penrose has in mind. Thus "For a quantum system where the number of basis states is greater than two, the space of physically distinguishable states is more complicated than the Riemann sphere. ... every spin state ... for spin $\mathrm{h} n / 4 \pi$ $\ldots$ is uniquely characterized by an (unordered) set of $n$ points on the Riemann sphere - i.e., by $n$ (usually distinct) directions outward from the centre ... There is something rather remarkable and puzzling about this description. One is frequently led to believe that, in some appropriate limiting sense, the quantum description of atoms (or elementary particles or molecules) will necessarily go over to classical Newtonian ones when the system gets large and complicated. However, just as it stands, this is simply not true. For, as we have seen, the spin states of an object of large angular momentum will correspond to a large number of points peppered all over the Riemann sphere. ... In general, quantum spin-states of large total spin are nothing whatever like classical ones." [p. 274]

${ }^{2}$ Fred Hoyle, in The Intelligent Universe (Holt, Rinehart and Winston, 1986), defines self-consistent "loops in time." Hoyle clearly has the idea that quantum vacuum zero-point fluctuations can be controlled from the future. He sees this as a sort of Shavian "life force" (Man and Superman) directing our evolution. "There can be few of us who have not wrestled mentally to a decision on some matter, only to act differently when the moment for implementing the decision actually arrives. This has the look of individual events in the brain. [p. 204] ... It seems to me that biological systems are able in some way to utilize the opposite time-sense in which radiation propagates from future to past. Bizarre as this may appear, they must somehow be working backwards in time. [p. 213] ... "At first sight communication from future to past seems to lead to logical inconsistency ... the less recognizable individual quantum events controlled from the future, as when we make up our minds to do one thing rather than another, can also have a major influence ... To avoid inconsistencies, both time senses must be linked into a consistent kind of loop. Properly speaking one should think in terms of loops in time, not in terms of cause and effect. ... The concept can be made clearer by an imaginary example, a thought experiment ... The reader who is trying to increase the amount of trouble in the world might say: Just give me the chance to go back in time... I will deliberately arrange that my ancestors don't meet ... By doing so he will destroy himself ... so the reader hesitates, trying to make up his mind, which he eventually does through an individual quantum event in the brain, an event which takes the form that preserves logical consistency ... believing himself to be acting voluntarily, whereas, he is really acting through a control from the future which always preserves consistency." [p. 247]

If Hoyle is right here, then Stapp et al. (e.g., Endnote 7) must be wrong. Hoyle continues: "If events could operate not only from past to future, but also from future to past, the seemingly intractable problem of quantum uncertainty could be solved. Instead of living matter becoming more and more disorganized, it could react to quantum signals from the future ... the information necessary for the development of life. [p. 213] ... the trouble we can now see with most of the fundamental questions about life and the origin of the Universe is that they are asked back-to-front. It is far less difficult to grapple with the issues in a futureto-past sense, because then we approach the ultimate cause instead of receding from it: the ultimate cause being a source of information, an intelligence if you like, placed in the remote future. [p. 214] .. Oxygen and carbon are like two radio receivers, each tuned to a particular wavelength. Unless the tunings are right ... far more oxygen is produced than carbon, but as it happens, the tunings are indeed correct ... The problem is to decide whether these apparently coincidental tunings are really accidental or not, and therefore whether or not life is accidental ... Could it be that the tunings ane intelligently deliberate? [p. 219] ... The list of Anthropic properties, apparent accidents of a non-biological nature without which carbon-based and hence human life could not exist, is large and impressive. [p. 219] ... Such properties seem to run through the fabric of the natural world like a thread of happy accidents. But there are so many of these odd coincidences essential to life that some explanation seems to be required to account for them." [p. 220].

Compare Hoyle to Henry Dwight Sedgwick almost eighty years earlier. Hoyle writes: "Instead of the Liniverse beginning in the wound-up state of the Big Bang, degenerating ener since, an initially primitive state of affairs could wind itself up gradually as time proceeds, becoming more, not less sophisticated, from past to future." Sedgwick wrote: "Things must be judged in the light of the oxering moming, not of the setting stars. It is not the past which, lik $\mathrm{an}$ unooling spring, pushes us on; creation faces the future, and is dram ansand by an irresistable attraction" ["House of Sorrow," Apologi for Old Maids (1908)]

${ }^{3}$ MS. Momis, Tip Thorne and Ulvi Yurtsever, Phys. Rev: Lett, 61, 1446 (26 Sept 1968) " Th the lass of physics permit an advanced civilization to creale and mintain a wormole in space for interstellar travel, then that wominle cae be crsened into a time machine with which causality might be riolastle ... What constraints do the laws of physics place on the activise of an atitrarily advanced civilization? ... We begin by abing wheter the las of physics permit an arbitrarily advanced civileston to arstret and maintain wormholes for interstellar travel. Such a worihte is a shen handle in the topology of space which links widely segures negins of the tniverse. ... Wormhole creation, with such mild sorrine candere ... must be accompanied by closed timelike curves Cre can ingegine an advanced civilization pulling a wormhole out of the 
analyzable by techniques now being developed for computation of spontaneous wormhole production by quantum tunneling. ... At $T=0$, the wormhole's mouths are at rest near each other. Subsequently, the left mouth remains at rest while the right mouth accelerates to near-light speed, then reverses its motion and returns to its original location. ... This motion causes the right mouth to age less than the left as seen by the exterior. Consequently, at late times by traversing the wormhole from right mouth to left, one can travel backward in time (i.e., one can traverse a closed timelike curve) and thereby, perhaps, violate causality." V.P. Frolov and I.D. Novikov, Phys. Rev. D 421057 (15 Aug. 1990): "It is shown that the interaction of a wormhole with the surrounding matter and with the external gravitational field almost inevitably transforms it into a time machine."

Kip S. Thorne and M.S. Morris, Am J. Phys. 56, 395 (1988): “The description touches base with Carl Sagan's novel, Contact, which, unlike most science fiction novels treats such travel in a manner that accords with the best 1986 knowledge of the laws of physics." I would like to record a bit of history here for the record. In the 1979 Oui article, "Science of the Impossible," Robert Anton Wilson describes my early efforts in designing a superluminal communication device. My ideas were known to Carl Sagan through various popular works like Space-Time and Beyond (E.P. Dutton, 1975) and Martin Gardner's MIT Technology Review article "Magic and Paraphysics" reprinted in Science, Good, Bad and Bogus (Prometheus). When Wilson asked what Sagan thought of my ideas, Sagan replied, "at most a playful notion." Indeed, Sagan playfully incorporated some of them in his novel Contact, which in turn was the catalyst for Thorne's discovery of the traversable wormhole solutions that require causality violation. For example, Thorne $\mathrm{et} \mathrm{al}$. write: "we adopt the spirit of Contact in which the protagonist and wormhole traveler is Dr. Ellie Arroway; she could emerge whenever she wishes: late in the evolution of the universe or, more interestingly, early enough to return to Earth and kill her own newborn mother (causality violation)." (p. 396) Thorne continues: "It is essential in these solutions that the wormhole possesses a throat at which there is no horizon; and this property ... places an extreme constraint on the material that generates the wormhole's curvature. In the wormhole's throat that material must possess a radial tension $\tau_{0}$ with the enormous magnitude $=$ pressure at the center of the most massive of neutron stars $\times 20 \mathrm{~km}^{2} /($ circumference of throat $)^{2}$. Moreover, this tension must exceed the material's density of mass-energy, $\rho_{0} c^{2}$. No known material has this $\tau_{0}>\rho_{0} c^{2}$ property ... However, it is not possible today to rule out firmly the existence of such material; and quantum field theory gives tantalizing hints that such material might, in fact, be possible. [p. 395] ... Sometimes one sees assertions that the speed with which signals should propagate in exotic material is $|\tau / \rho|^{1 / 2}$ which exceeds the speed of light ... [p. 406]." J. Friedman, M.S. Morris, F. Echeverria, G. Klinhammer, K. Thorne, and I.D. Novikov, Phys. Rev. D 42, 1915 (15 Sept. 1990): "The only type of causality violation that the authors would find unacceptable is that embodied in the science fiction concept of going backward in time and killing one's younger self (changing the past). Some years ago one of us (Novikov) briefly considered the possibility that CTC's [closed timelike curves] might exist and argued that they cannot entail this type of causality violation: Events on a CTC are already guaranteed to be selfconsistent, Novikov argued; they influence each other around the closed curve in a self-adjusted, cyclical, self-consistent way. The other authors have arrived at the same viewpoint. [p. 1916] ... The simplest way to impose the principle of self-consistency in quantum mechanics (in a classical space-time) is by a sum-over-histories formulation in which one includes all those, and only those, histories that are self-consistent. ... We suspect that for any quantum system in a classical wormhole with a stable Cauchy horizon, the sum over all self-consistent histories will give unique, self-consistent probabilities for the outcomes of all sets of measurements that one might choose to make. ... A recurring theme in this paper is the conclusion that CTC's may not be as nasty as people have assumed. This conclusion is reinforced by an analysis of global conservation laws in asymptotically flat wormhole space times with CTC's. It is shown that, just as without CTC's, so also with them, all differential conservation laws expressed by the vanishing divergence of a vector field (e.g., the conservation of charge and baryons, and in stationary spacetimes the conservation of energy) give rise to global conservation laws. When CTC's are present, these global laws express the constancy of the sum of three quantities: (i) the amount of the given item that is present in the Universe at a specific moment of time, plus (ii) the amount that is temporarily absent by virtue of time traveling to the future through a wormhole, minus the amount that is temporarily doubly present because time traveled to the past through the wormhole. It seems reasonable to hope that, in wormhole spacetimes with CTC's, the sum-over-histories formulation of quantum mechanics will give rise to this type of conservation law for probabilities. However, thus far we have not been able to verify that this is so. [p. 1918] ... any being who went through a wormhole and tried to change the past would be prevented by physical law from making the change; i.e., the 'free will' of the being would be constrained. Although this constraint has a more global character than constraints on free will that follow from the standard local laws of physics, it is not obvious to us that this constraint is more severe than those imposed by standard physical law." [p. 1928]

See Endnote 2 (Hoyle) for the origin of the idea of global selfconsistency on CTC's.

An alternative equivalent picture is that CTC's connect different parallel universes which are like sheets of a Riemann surface. That is, the CTC's go through the branch cut. The condition that a complex function of a complex variable be single-valued on the multisheeted Riemann surface is analogous to the global condition of causal self-consistency along CTC's traversing parallel universes. Parallel universes are more like different cross sections of a fiber bundle over a single base space. The extradimensional cross sections above the base space represent different patterns of mutually consistent measurements. These parallel world patterns include the split consciousness of observer-participators. Therefore, an observer-participator confined to a single consciousness track will see equipment malfunction in order to avoid "killing one's past self" type of paradoxes. I independently thought of using self-consistent sums-overhistories as now advocated by Thorne et al.

I have conjectured that global self-consistency also causes automatic equipment to malfunction at-a-distance if that equipment is part of an anomalous loop in time. This would be the basis of a new kind of "psychotronic" weapon (to use a term popular in the pre-glasnost Soviet Union and Eastern Europe). There are unsubstantiated rumors of UFO's jamming our air defenses and hovering over our nuclear weapon sites with impunity, for example, Omni (Dec. 1990) and Barry Greenwood, Clear Intent (CAUS Fawcett, 1984). If these reports were true, and I am 
not saying that they are because I have no way of knowing (indeed Edward Teller emphatically says UFO's do not exist even though Moscow also says they do not exist, so that the present example is in the spirit of an amusing exercise), then it would be evidence that UFO's are time travel devices involving CTC's, since some attempts to destroy them would involve a causal paradox and would necessarily fail. Even if we totally discount UFO reports as Teller thinks we should, and he is in a better position to know the facts, nevertheless, the new work on traversable wormholes requires a fresh look at the SETI problem.

${ }^{4}$ C.L. Bennett, Phys. Rev. A 35, 2409 (1987): "Evidence has been discovered for the violation of microscopic causality in the Compton scattering of light by protons. ... and photomeson production cross sections throughout the region of the $\Delta(1232)$ resonance. ... Macroscopic relativistic causality is the condition that energy not be transmitted at speeds exceeding $c$. . . this condition is obeyed by Compton scattering even with preacceleration. Microscopic relativistic causality is the condition that a response strictly vanish at at time and place prior to a stimulus. ... this condition is not true for classical Compton scattering, or for Heitler's expression ... when we speak of generic causality violation, we mean, specifically, microscopic relativistic causality violation. ... it is possible to obey the dispersion relations, yet violate macroscopic relativistic causality ... It is possible to obey the dispersion relations, obey macroscopic relativistic causality, but disobey microscopic relativistic causality. ... It is possible to violate the dispersion relations, obey macroscopic relativistic causality, but not obey microscopic relativistic causality."

Ibid. 2420: "We have presented a simple semiclassical model of precausality, which fits the proton Compton scattering and photo absorption data better than any dispersion theory. We also suggest that because of the possible overestimate of systematic errors in the best existing measurement of the total photoabsorption cross section, previous agreements with phenomenological models and mild disagreements with dispersion relations may become significant disagreements. We conclude that there is significant evidence for the existence of precausal behavior in nature." Ibid. 36, 4139 (1987): “. . radiative reaction terms responsible for microscopic causality violation ... results from the absence of intrinsic self-interaction ... The apparent self-force of radiative reaction ... is seen to be no more than the superposition of the advanced influences of all the other particles in the environment. ... the time evolution of a state is affected by the future as well as the past [p. 4142] ... spontaneous emission is understood as the interaction with remote particles via advanced waves from the future. Spontaneous emission only appears to us macroscopic forward-in-time-bound observers as spontaneous, since we are not able to control the advanced future interactions with remote particles. The best we can do is follow the illusory quantum to its ultimate absorption, in order to discover which particle of the future interacted with our apparent spontaneous emitter. ... If the decay rate of an excited state is very small, then the incidence of interactions with the future is correspondingly less frequent." [p. 4144]

See Endnote 2 (Hoyle) for support of the idea that individual quantum events like quantum fluctuations can be controlled from the future. Y. Ne'eman, Proc. Natl. Acad. Sci. U.S.A. 80, 7051 (1983): "in the geometry of a fiber bundle describing a gauge theory, curvature and parallel transport ensure and impose nonseparability. ... Such a manifold ... corresponds to a nontrivial juxtaposition of a (vertical) group space (or group representation space), the fiber at each point of the (horizontal) base space (generally space-time). ... the geometry of fiber bundles ... provides ... a classical geometrical realization of EPR nonlocal action or nonseparability of the composite wave function. ... in a fiber bundle geometry, the manifold is constrained so as to preserve parallelism whatever the magnitude of the base space interval. ... Local gauge invariance implies local unobservability of a variable. A measurement or an alteration at $x$ is locally unobservable, but the constraints on the geometric structure of the fiber bundle do transmit the relevant information over the entire base space All points of the base manifold thus 'know' of the alteration at $x$, even though it cannot be known at $x$ itself."

${ }^{6}$ J.F. Clauser and A. Shimony, Rep. Prog. Phys. 41, 1881 (1978): "Bell's theorem represents a significant advance in understanding the conceptual foundations of quantum mechanics. The theorem shows that essentially all local theories of natural phenomena that are formulated within the framework of realism may be tested using a single experimental arrangement. Moreover, the predictions by these theories must significantly differ from those by quantum mechanics. Experimental results evidently refute the theorem's predictions for those theories and favor those of quantum mechanics. The conclusions are philosophically startling: either one must totally abandon the realistic philosophy of most working scientists, or dramatically revise our concept of space-time." See, also, J.S. Bell, Physics 1, 195 (1964).

The literature on no-EPR-faster-than-light-communication proofs is extensive, for example, Pagels, The Cosmic Code (Simon and Shuster, 1982), Chaps. 12, 13. A. Shimony, Proceedings of an International Symposium on the Foundations of Quantum Mechanics (Tokyo, 1983), p. 225. P. Eberhard, Nuovo Cimento 46B, 392 (1978). G.C. Ghirardi and T. Weber, Lett. Nuovo Cimento 26, 599, (1979). G.C. Ghirardi, A. Rimini, and T. Weber, Lett. Nuovo Cimento 27, 294 (1980). Their key assumption, which I deny, is that a measurement on one member of the quantum connected pair always involves an interaction which does not affect the "far-lying system" and apparatus. This implies that the one-sided measurement interaction is an operator only of the Hilbert space of the measured system plus local apparatus. This further implies that the one-sided measurement process can be described by a unitary operator which is an identity operator on the Hilbert space of the other side. My claim is that the Feynman rules deny all this for a clever enough experimental design.

H.P. Stapp, Nuovo Cimento 40B, 191 (1977). This paper does not present a proof against FTL signals Quite the contrary, we find ambiguous and highly suggestive remarks like, The central mystery of quantum theory is 'how does information grt around so quick? How does the particle know that there are tor slit? How does the information about what is happening everywhere ele gre collected to determine what is likely to happen here? Hos does the particle know that it was looked for in some far-zasy place and not found? Quantum phenomena provide prima facie evidence the irformation gets around in ways that do not conform u descicl ites. Thus, the idea that information is transferred superluminaly ix. a proin, not unreasonable. Everything we know about Nature is in acuri with the idea that the fundamental process of Nature lies ouside spactine (surveys the space-time continuum globally), but generates ees the are located in space-time. The theorem of this paper suppors tis re $\alpha$ Kature by showing that superluminal transfer of infirmane is neossary barring certain alternatives ... that seem less nondk: [z 201] 
Stapp later clarified his position by explicitly forbidding controlled nonlocality (i.e., useful practical FTL signaling of messages) in, Found. Physics, 10, 791 (1980): "The failure of the locality property in ... quantum theory ... does not contradict Einstein's principle that no signal travels faster than light. For by a signal is meant a controllable transfer of information - a message. Within the structure of these formalisms no such controlled faster-than-light information transfer is possible. This follows immediately from the fact that whereas within the quantum formalism (or the classical formalism) the probability of a specified result in one region, subject to the condition of a specified result in the other region, depends in general on the latter specification, and hence on the experimental setting in the other region, nevertheless, a summation over all possible results of the experiment in the other region with proper weights gives a result that is independent of the choice of the experimental setting in that region. This entails that there is no predictable dependence of the observations in one region upon the choice of setting in the other."

P.J. Bussey, Phys. Lett. 90 A, 9 (1982). Bussey's proof is like Stapp's first objection. It does not include the possibility of interference.

P.H. Eberhard and R.R. Ross, Found. Phys. Lett. 2, 127 (1989). I agree with Eberhard's conclusions that quantum field theory as presently formulated with the subluminal mass shell only, the Feynman causal boundary conditions, and no-violation of the spin-statistics connection will not permit superluminal communication of the second kind. However, I do not think they have eliminated superluminal communication of the third kind within the framework of the conventional quantum mechanics of photons.

D. Dieks, Phys. Lett. 92A, 271 (1982): "We demonstrate that such superluminal communication is not possible. The crucial role of the linearity of the quantum evolution laws in preventing causal anomalies is stressed." This is a refutation of Herbert's "FLASH" (see Ref 3).

W.K. Wooters and W.H. Zurek, Nature 299, 802 (1982).

${ }^{8}$ E. Kramer, The Growth of Modern Mathematics (Hawthorne, 1970), p. 402: "The new idea ... was that all the various species of Geometry which, during the 19th century, in particular, had multiplied exceedingly (metrical geometry, projective geometry, line geometry, etc.) could be regarded from a single standpoint, that of the Theory of Groups, each different geometry being conceived as the theory of the invariants of an appropriate group."

9 Rudy Rucker, Infinity and the Mind, "Conversations with Godel" (Birkhauser, 1982), p. 168. Rucker quotes Godel: "time-travel is possible, but no person will ever manage to kill his past self. The a priori is greatly neglected. Logic is very powerful."

10 W. Pauli, Theory of Relativity (Pergamon, 1958), p. 16: "Suppose it were possible that an effect in system $\mathrm{K}$ is propagated with velocity greater than $c$. There would then exist a system $K^{\prime}$ (moving relative to $K$ with velocity less than $c$ ) in which an event causing another, later event in $\mathrm{K}$ would happen after it. ... This would be tantamount to upsetting the concepts of cause and effect, and it can therefore be concluded that it is impossible to send out signals with a velocity greater than that of light." Pauli quotes Einstein, Ann. Phys. Leipzig. 23, 371 (1907). See, also, W.K.H. Panofsky and M. Phillips, Electricity and Magnetism (AddisonWesley, 1962), p. 302: "If it were possible to make $\left(d t^{\prime} / d t\right)<0$ by a suitable choice of $u$, then the temporal sequence of two events would be reversed between the two frames under consideration. This would be a logical contradiction if (a) the two events represent cause and effect, and (b) the sense of time has an invariant significance ... we are forced to conclude that, in order for the sequence between cause and effect to be preserved in all frames, in any particular frame $u<c$." See, also, H. Schmidt, Found. Phys. 8, 463 (1978). The Princeton University PEAR Laboratory directed by Robert Jahn reports successful "precognitive remote viewing," and Penrose quotes EEG data suggesting that free will requires retroactivity from controlled future delayed free choice to past action (which is a prior effect of that freely willed choice). On "powerful instances of causality violating phenomena" (Ref. 4), see, also, I.J. Good, The Scientist Speculates (Capricorn, 1965), p. 314. P. Fitzgerald, Philosophia 4, 513 (1974). C.W. Rietdiik, Physica B 151, 358, (1988). Rietdijk claims (p. 359) that superluminal transport of angular momentum violates rotational symmetry.

11 Yakir Aharonov, Jeeva Anandan, Sandu Popecu, and Lev Vaidman, Phys. Rev. Lett. 64, 2965 (1990). "We introduce a new notion: a superposition of time evolutions (rather than that of states) ... a particular superposition of time evolutions with the same Hamiltonian but different periods of time is equivalent to a time evolution for a very different (possibly even negative) period of time. This provides an example of a new type of 'time-translation machine' which is peculiar to quantum systems and has no classical analog. ... This machine will succeed to work only very rarely."

12 S. Hawking, A Brief History of Time (Bantam, 1988), p. 134: "One must add up the waves for particle histories that are not in the 'real' time that you and I experience but take place in what is called imaginary time. ... This has an interesting effect on space-time: the distinction between time and space disappears completely."

13 L.D. Faddeev and A.A. Slavnov, Gauge Fields (Benjamin Cummings, 1982), p. 98: "The expression ... for the $S$-matrix contains the nonlocal functional det $M \ldots$ we may, however, use for det $M$ the integral expression ... where $c(x)^{*}$ and $c(x)$ are anticommuting scalar functions (generators of the Grassmann algebra) ... At the cost of introducing the fictitious fields $c^{*}, c$ we have succeeded in taking the relativity principle into account in such a way that the $S$-matrix is represented in the form of an integral of $\exp \{i$ Action $\} ..$ The fictitious particles have no corresponding external lines and enter into the $S$-matrix only by means of closed loops. ..." [p. 132] "... the manifestly covariant gauges, such as the Lorentz one, for which ... renormalizability is obvious. However, in the Lorentz gauge we cannot construct a Hamiltonian ... therefore the unitarity of the $S$-matrix is not obvious. From the viewpoint of the operator formalism the $S$-matrix in the Lorentz gauge acts in the 'big' space containing both physical and non-physical states (longitudinal and 'time' photons, scalar fermions, Goldstone bosons) and, generally speaking, is unitary only in this space, in which the metric is indefinite. The unitarity of the $S$-matrix in the physical subspace, the states of which correspond to fields of matter and to transverse vector quanta, is a consequence of the relativity principle, according to which all observable are independent of the gauge conditions actually chosen." Note, also, p. 135 Eqs. $(3,11)$, (3.12) the introduction of both scalar fermions and spinor bosons in Pauli-Villars invariant regularization of Yang-Mills gauge theory.

14 W. Pauli, Phys. Rev. 58, 716 (1940); Idem Prog. Theor. Phys. 5, 526 (1950). K. Svozil, Phys. Rev. A 39, 2222 (1989): "Local causality is assured by a proper connection between spin and statistics." V.B. Berestetskii, E.M. Lifshitz, and L.P. Pitaevskii, Quantum Electrodynamics (Perga- 
mon, 1982), p. 914. It is shown that the energy of spinor boson fields is not positive definite. This would allow propagation of negative energy forwards in time, that is, vacuum instability.

15 "Van Horne talked about himself, his hopes of finding a loophole in the second law of thermodynamics. There bas to be one and it's the same ... loophole whereby everytbing crossed over from nonbeing. It's the singularity at the bottom of the Big Bang ... but Suky baby ... it's a force ... that operates across space instantly and has notbing to do with the electromagnetic field... There's a formula out there ... and it's going to be as elegant as good old $E=m c^{2}$. The sword from the stone. You know what I mean ... You gotta swing those electrons ... You know what Cooper pairs are ... that make up the beart of superconductors... There's no stopping us Suky, babee. Suky laughed ... why couldn't a wild man ... blunder into one of the Universe's secrets?" John Updike, The Witches of Eastwick (p. 134). See, also, Updike's Roger's Version for an entertaining exposition of the argument from design according to the anthropic cosmological principle.

R.W. Sperry, Psychol. Rev. 76, 532 (1969). Stapp quotes the remark by Sperry in, Found. Phys. 15, 42 (1985). Stapp argues that the brain has a macroscopic quantum wave function and that the experience of feeling consciousness is associated with the collapse of the wave function in the transition of possibility to actuality. "The wave function of the brain ... will have parts representing all of the conceivable possibilities that could emerge in the given situation due to variations in, for example, the times and rates of the penetrations of all the potential barriers associated with all the chemical processes occurring in the brain ... one can then identify the cortical patterns of excitation that initiate perceptually different responses as different branches of the wave function of the brain .... a reduction must occur before the perceptibly different course of action comes into being. A choice among the branches of the wave function that initiate these perceptibly different alternative possible courses of action would be, functionally, a choice among these different possible courses of action. It would be represented in quantum theory by a reduction of wave packet to the corresponding branch. The act of choosing one of these courses of action may then be identified as both a psychical and physical act: the subjectively felt act of selecting a course of action may be represented in the physicist's construction of physical reality by the reduction of wave packet that selects this course of action. Thus what is 'felt' at the psychical level is identical to what 'happens' at the physical level." [p. 39] I agree with that. However, Stapp is inconsistent later on in the paper, because he tries to hold on to the Born $|\psi|^{2}$ probability interpretation for these complex mental reductions. This contradicts Stapp's view that quantum theory allows human free will and responsibility.

As is well known in philosophical discussions of the free will problem, not only does the absolute determinism of classical mechanics forbid responsible free will, but the alleged absolute randomness of quantum process also does. Stapp holds on to macroscopic causality when he says that consciousness now depends upon past conscious acts. I would also add that it also depends upon future conscious acts. The Born $|\psi|^{2}$ probability interpretation must be a limiting case for minimal complexity of repeatable individual quantum events that permit realizable actual ensembles in the phenomena. Complex mental acts of high level creation of cultural works, for example, a Mozart opera, an Einstein theory of relativity, or a poem by Coleridge, are not repeatable and not susceptible to an ensemble probability interpretation, without throwing the baby out with the bath water, if we wish to have responsible free choice. That is, we must be able to choose into which branch we want to collapse the brain wave function, to make an analogy with low complexity quantum theory. For an unpolarized beam, not only can we choose to measure spin along the $z$ direction, we can also choose to have the spin eigenvalue $+1 / 2$ over and over again in violation of the Born interpretation and the Feynman rules. If this is so for high complexity macroscopic quantum processes, then we have a new form of nonlinear controlled nonlocality that permits superluminal and retroactive "telepathy" and "psychokinesis." That is, if we follow Stapp's view on responsible free choice in a quantum universe to its logical conclusion, we must be prepared to throw out the statistical predictions of quantum theory as a low complexity approximation transcended by intelligent quantum systems.

\section{References}

1. N.D. Mermin, Am. J. Phys. 58, 731 (1990).

2. Idem, Phys. Today (June 1990).

3. B. Josephson and F. Pallikari-Viras, Found. Phys. 21, 197 (1991).

4. M.A. Horne, A. Shimony, and A. Zeilinger, Phys. Rev. Lett. 62, 2209 (1989).

5. Y. Aharonov, D.Z. Albert, and L. Vaidman, Phys. Rev. D 34, 1805 (1986).

6. N. Herbert, Found. Phys. 12, 1171 (1982).

7. C.C. King, Phys. Essays 2, 128 (1989).

8. K. Svozil, "What's Wrong with SLASH?", Technical University of Vienna, 1989, unpublished preprint.

9. H. Woolf, editor, Some Strangeness in the Proportion (Addision Wesley, 1980), p. 481.

10. Y. Aharonov and D. Bohm, Phys. Rev. 122, 1649 (1961).

11. A. Pais, Inward Bound (Oxford, 1986), p. 499. J.S. Toll, Phys. Rev. 104, 1760 (1956). M. Gell-Mann, M.L. Goldberger, and W.E. Thirring, Phys. Rev. 95, 1612 (1954). S. Mandelstam, Rep. Prog. Phys. 2599 (1962).

12. O.M. Bilaniuk and E.C.G. Sudarshan, Phys. Today, 43 (May 1969).

13. (a) Optics Guide 5 (Melles Griot, 1990), Secs. 4-5; (b) ibid, Secs. 4-7.

14. W.K.H. Panofsky and M. Phillips, Electricity and Magnetism (AddisonWesley, 1962).

15. H.P. Stapp, Found. Phys, 10, 791 (1980).

\section{Jack Sarfatti}

Hebrew Academy of San Francisco

P.0. Box 26548

San Francisco, California 94126 U.S.A. 